\title{
Fate of the conformal order
}

\author{
Alex Buchel \\ Department of Applied Mathematics, Department of Physics and Astronomy, University of Western \\ Ontario, London, Ontario N6A 5B7, Canada and Perimeter Institute for Theoretical Physics, \\ Waterloo, Ontario N2J 2W9, Canada
}

(Received 24 November 2020; accepted 24 December 2020; published 11 January 2021)

\begin{abstract}
We use holographic correspondence to study transport of the conformal plasma in $\mathbb{R}^{2,1}$ in a phase with a spontaneously broken global $\mathbb{Z}_{2}$ symmetry. The dual black branes in a Poincare patch of asymptotically $\mathrm{AdS}_{4}$ have "hair" - a condensate of the order parameter for the broken symmetry. This hair affects both the hydrodynamic and the nonhydrodynamic quasinormal modes of the black branes. Nonetheless, the shear viscosity of the conformal order is universal, the bulk viscosity vanishes and the speed of the sound waves is $c_{s}^{2}=\frac{1}{2}$. We compute the low-lying spectrum of the nonhydrodynamic modes. We identify a quasinormal mode associated with the fluctuations of the $\mathbb{Z}_{2}$ order parameter with the positive imaginary part. The presence of this mode in the spectrum renders the holographic conformal order perturbatively unstable. Correspondingly, the dual black branes violate the correlated stability conjecture.
\end{abstract}

DOI: 10.1103/PhysRevD.103.026008

\section{INTRODUCTION AND SUMMARY}

Following the general suggestion of [1], we proposed in $[2]^{1}$ a holographic model for a conformal order ${ }^{2}$ : a thermal phase of a conformal gauge theory in $\mathbb{R}^{2,1}$ with a nonzero expectation value of an irrelevant, dimension $\Delta=4$ operator $\mathcal{O}$, spontaneously breaking the global $\mathbb{Z}_{2}$ symmetry. Specifically, for a model $S_{\mathrm{CFT}_{3}^{\psi}}$ in [2] two distinct thermal phases were identified:

$$
\begin{aligned}
\frac{\hat{\mathcal{F}}}{(\pi T)^{3}} \equiv & \frac{384}{c} \frac{\mathcal{F}}{(\pi T)^{3}}=-\frac{64}{27} \\
& \times \begin{cases}1, & \langle\mathcal{O}\rangle=0 \Rightarrow \mathbb{Z}_{2} \text { is unbroken; } \\
\kappa(b), & \langle\mathcal{O}(b)\rangle \neq 0 \Rightarrow \mathbb{Z}_{2} \text { is broken, }\end{cases}
\end{aligned}
$$

where $\mathcal{F}$ is the free energy density, $T$ is the temperature, and $c$ is the central charge. The constant $0 \leq \kappa \leq 1$ and the thermal expectation value of $\mathcal{O}$ (in the symmetry breaking phase) depends on the parameter $b$ of the dual gravitational action $^{3}$ :

${ }^{1}$ See also [3].

${ }^{2}$ See [4] for related nonconformal models.

${ }^{3}$ We set the radius of an asymptotic $\mathrm{AdS}_{4}$ geometry to unity.

Published by the American Physical Society under the terms of the Creative Commons Attribution 4.0 International license. Further distribution of this work must maintain attribution to the author(s) and the published article's title, journal citation, and DOI. Funded by SCOAP ${ }^{3}$.

$$
S=\frac{c}{384} \int d x^{4} \sqrt{-\gamma}\left[R+6-\frac{1}{2}(\nabla \chi)^{2}-2 \chi^{2}-b \chi^{4}\right] .
$$

The symmetry broken phase exists only $b<b_{\text {crit }, 0} \equiv-\frac{3}{2}$. Note that since the specific heat density $c_{v}$ is

$$
\begin{aligned}
c_{v} \equiv & -T\left(\frac{\partial^{2} \mathcal{F}}{\partial T^{2}}\right)_{v}=\frac{c \pi^{3} T^{2}}{27} \\
& \times \begin{cases}1, & \mathbb{Z}_{2} \text { is unbroken } ; \\
\kappa(b) \geq 0, & \mathbb{Z}_{2} \text { is broken, }\end{cases}
\end{aligned}
$$

it is positive, irrespectively whether or not the global symmetry $\mathbb{Z}_{2}$ is broken.

In this paper we continue the study of the model (2). First, we point out that there are multiple branches, indexed by $i=0,1, \ldots$, of the "hair" - the thermal expectation values of $\mathcal{O}$. In the holographic dual, the index is related to the number of zeros in the radial profile of the holographic bulk scalar $\chi$. For two branches with $i<j$,

$$
\kappa_{i}(b)>\kappa_{j}(b), \quad b \in\left(-\infty, b_{\text {crit }, j}<b_{\text {crit }, i}\right],
$$

i.e., the branches with the higher index are increasingly less thermodynamically favored-all the symmetry breaking phases have a higher free energy density than that of the $\mathbb{Z}_{2}$-symmetric phase. We find that the thermodynamics of all the symmetry broken phases resemble that of the symmetric phase in the limit $b \rightarrow-\infty$,

$$
\left(1-\kappa_{i}(b)\right) \propto+\frac{1}{(-b)}, \quad\langle\mathcal{O}\rangle_{i} \propto \frac{1}{\sqrt{-b}} .
$$


Notice that the gravitational potential for $\chi$

$$
V \equiv 2 \chi^{2}+b \chi^{4}
$$

is unbounded from below as $b<0$, (naively) implying that the exotic features of the model (2) are due to this "sickness." This is not the case: given (5), it is clear that a simple deformation of the scalar potential, i.e.,

$$
V \rightarrow V_{g}=V+g \chi^{6}, \quad g>0,
$$

makes it bounded, while not affecting the thermodynamics of the model, at least as $b \rightarrow-\infty$ :

$$
V \propto \frac{1}{b}, \quad V-V_{g}=g \chi^{6} \propto \frac{1}{b^{3}} .
$$

We explicitly demonstrate that the symmetry breaking phases at finite $b$ are robust against the deformation (7), for small enough $g>0$.

We study the coupled metric-scalar fluctuations in the symmetry broken phases of (2) and compute the spectrum of the quasinormal modes (QNMs) of the black branes dual to the conformal order on the lowest branch. ${ }^{4}$ As expected from the conformal theory, irrespectively of the symmetry breaking, we find

$$
c_{s}^{2}=\frac{1}{2}, \quad \zeta=0
$$

for the speed of the sound waves $c_{s}$ and the bulk viscosity $\zeta$ correspondingly. From the dispersion relation of the hydrodynamic mode in the shear channel [5] we recover the universal result [6-8] for the ratio of the shear viscosity $\eta$ to the entropy density $s$

$$
\frac{\eta}{s}=\frac{1}{4 \pi}
$$

Besides the sound wave- a hydrodynamic QNM in the scalar channel [5] - there are two branches of the nonhydrodynamic modes coming from the mixing of the helicity zero metric fluctuations in the equilibrium black brane geometry and the gravitational bulk scalar field, whose boundary values determine the order parameter for the $\mathbb{Z}_{2}$ symmetry breaking. There are two purely dissipative nonhydrodynamic modes ${ }^{5}$ :

$$
\operatorname{Re}[\mathfrak{w}]=0, \quad \operatorname{Im}[\mathfrak{w}] \neq 0
$$

\footnotetext{
${ }^{4}$ We expect that the conclusions apply for the $i>0$ branches of the conformal order as well.

${ }^{5}$ We take the space-time dependence of the QNM fluctuations to be $\propto e^{-i \omega t+i \vec{k} \vec{x}}$ and introduce $\mathfrak{w}=\frac{\omega}{4 \pi T}$ and $\mathfrak{q}=\frac{|\vec{k}|}{4 \pi T}$.
}

One of these QNMs, $\mathfrak{w}_{u}(\mathfrak{q})$, at least when the spatial momentum $\mathfrak{q}$ is below some critical value $\mathfrak{q}_{c}=\mathfrak{q}_{c}(b)$, has a positive imaginary part, i.e.,

$\operatorname{Re}\left[\mathfrak{w}_{u}(\mathfrak{q})\right]=0, \quad \operatorname{Im}\left[\mathfrak{w}_{u}(\mathfrak{q})\right]= \begin{cases}\geq 0, & \mathfrak{q} \leq \mathfrak{q}_{c} \\ <0, & \mathfrak{q}>\mathfrak{q}_{c}\end{cases}$

We further show that

$$
\operatorname{Im}\left[\mathfrak{w}_{u}(\mathfrak{q}=0)\right]>0, \quad \text { as } b \in\left(-\infty, b_{\text {crit }, 0}\right),
$$

approaching zero in the limit $b \rightarrow b_{\text {crit, }, \text {. The presence of }}$ this mode in the spectrum implies that the translational invariant horizon of the black brane dual to a conformal order is perturbatively unstable to clumping. We expect ${ }^{6}$ that the dynamical evolution of the perturbed conformal order will result in a destruction of the ordered phase, with the $\mathbb{Z}_{2}$ symmetric equilibrium phase being the attractor. We show that the unstable QNM is present on the higher branches of the conformal order as well.

Note that the perturbative instability of the holographic conformal order proposed in [2] implies that the correlated stability conjecture of $[9,10]$ for the dual black branes is violated: while these black branes have positive specific heat, they are dynamically unstable. ${ }^{7}$ We stress that the positive specific heat is not the same as the thermodynamic stability, tacitly assumed in [9] - the latter concept typically applies to the thermodynamically dominant phases (having the minimal free energy density in the canonical ensemble), which is not necessarily the case. Indeed, unlike the ordered thermodynamically stable phases discovered in [1], the holographic conformal ordered phases in [2] are metastable, see (1).

A challenge remains to find an example of a stable holographic thermal conformal order-a phase of the black branes which is both the dominant one in the canonical ensemble, and is perturbatively stable with respect to the linearized fluctuations.

\section{HOLOGRAPHIC THERMAL CONFORMAL ORDER}

In this section we review the construction of the holographic conformal order proposed in [2] and discuss two generalizations:

(i) we show that there are multiple branches of the conformal order;

(ii) we show that the effective scalar potential in the gravitational dual can be made bounded, without affecting the existence of the ordered phases.

\footnotetext{
${ }^{6}$ Work in progress.

${ }^{7}$ See $[11,12]$ for other examples of the CSC violation.
} 


\section{A. Branches of the conformal order}

The starting point is the effective action (2). The thermal ordered states are dual to black brane solutions

$$
d s_{4}^{2}=-c_{1}^{2} d t^{2}+c_{2}^{2}\left[d x_{1}^{2}+d x_{2}^{2}\right]+c_{3}^{2} d r^{2},
$$

where all the metric warp factors $c_{i}$ as well as the bulk scalar $\chi$ are functions of the radial coordinate $r$,

$$
r \in\left[r_{0},+\infty\right),
$$

where $r_{0}$ is a location of a regular Schwarzschild horizon, and $r \rightarrow+\infty$ is the asymptotic $\mathrm{AdS}_{4}$ boundary. Introducing a new radial coordinate

$$
x \equiv \frac{r_{0}}{r}, \quad x \in(0,1]
$$

and denoting

$c_{1}=r\left(1-\frac{r_{0}^{3}}{r^{3}}\right)^{1 / 2} a_{1}, \quad c_{2}=r, \quad c_{3}=\frac{1}{r}\left(1-\frac{r_{0}^{3}}{r^{3}}\right)^{-1 / 2} a_{3}$,

we obtain the following system of ODEs (in a radial coordinate $\left.x,{ }^{\prime}=\frac{d}{d x}\right)$ :

$$
\begin{aligned}
& 0=a_{1}^{\prime}-\frac{3 a_{1}\left(a_{3}^{2}-1\right)}{2 x\left(x^{3}-1\right)}+\frac{1}{8} x a_{1}\left(\chi^{\prime}\right)^{2}+\frac{a_{3}^{2} a_{1} V}{4 x\left(x^{3}-1\right)}, \\
& 0=a_{3}^{\prime}+\frac{1}{8} x a_{3}\left(\chi^{\prime}\right)^{2}+\frac{3 a_{3}\left(a_{3}^{2}-1\right)}{2 x\left(x^{3}-1\right)}-\frac{a_{3}^{3} V}{4 x\left(x^{3}-1\right)}, \\
& 0=\chi^{\prime \prime}+\left(\frac{a_{1}^{\prime}}{a_{1}}-\frac{a_{3}^{\prime}}{a_{3}}+\frac{x^{3}+2}{x\left(x^{3}-1\right)}\right) \chi^{\prime}+\frac{\partial V a_{3}^{2}}{\left(x^{3}-1\right) x^{2}},
\end{aligned}
$$

where the scalar potential $V$ is given by (6), and $\partial V \equiv \frac{\delta V}{\delta \chi}$. Notice that $r_{0}$ is completely scaled out from all the equations of motion. Equations (18)-(20) have to be solved subject to the following asymptotics:

(i) in the UV, i.e., as $x \rightarrow 0_{+}$,

$$
\begin{aligned}
a_{1} & =1+a_{1,3} x^{3}+\mathcal{O}\left(x^{6}\right), \\
a_{3} & =1-a_{1,3} x^{3}+\mathcal{O}\left(x^{6}\right), \\
\chi & =\chi_{4} x^{4}+\mathcal{O}\left(x^{7}\right) ;
\end{aligned}
$$

(ii) in the IR, i.e., as $y \equiv 1-x \rightarrow 0_{+}$,

$$
\begin{aligned}
& a_{1}=a_{1,0}^{h}+\mathcal{O}(y), \quad a_{3}=\sqrt{\frac{6}{6-V\left(c_{0}^{h}\right)}}+\mathcal{O}(y), \\
& \chi=c_{0}^{h}+\mathcal{O}(y),
\end{aligned}
$$

where $V\left(c_{0}^{h}\right)$ implies that the scalar potential (6) is evaluated on the horizon value of the bulk scalar $\chi$.

In total, given $b$, the asymptotic expansions are specified by four parameters

$$
\left\{a_{1,3}, \chi_{4}, a_{1,0}^{h}, c_{0}^{h}\right\}
$$

which is the correct number of parameters necessary to provide a solution to a system of a single second-order and two first-order equations, $1 \times 2+2 \times 1=4$. It is straightforward to extract the thermodynamics of the resulting black brane:

$$
\begin{gathered}
\mathcal{F}=-\frac{c}{192}(\pi T)^{3} \kappa, \quad \mathcal{E}=-2 \mathcal{F}, \quad \frac{\langle\mathcal{O}\rangle}{c T^{4}} \propto\langle\hat{\mathcal{O}}\rangle=\chi_{4}, \\
T=\frac{r_{0}}{8 \pi} a_{1,0}^{h} \sqrt{36-6 V\left(c_{0}^{h}\right)}, \quad \kappa=\frac{36}{\left(a_{1,0}^{h}\right)^{2}\left(36-6 V\left(c_{0}^{h}\right)\right)},
\end{gathered}
$$

for the free energy density $\mathcal{F}$, the energy density $\mathcal{E}$, the temperature $T$, and the thermal expectation value of the conformal order $\langle\hat{\mathcal{O}}\rangle$. We explicitly indicated the $r_{0}$ dependence - all the parameters in (23), as well as $\kappa$ and $V\left(c_{0}^{h}\right)$ depend only on $b$.

From (24), the speed of the sound waves in the holographic conformal order plasma is

$$
c_{s}^{2}=-\frac{\partial \mathcal{F}}{\partial \mathcal{E}}=\frac{1}{2} .
$$

Additionally, since that temperature $T$ depends on $r_{0}$, and the horizon value of the scalar is $r_{0}$ independent,

$$
\frac{d}{d T}\left(\left.\chi(x)\right|_{x \rightarrow 1_{-}}\right)=\frac{d}{d T}\left(c_{0}^{h}\right)=0
$$

implying that the bulk viscosity of the corresponding plasma must vanish ${ }^{8}[15]$

$$
\frac{\zeta}{T^{2}}=0
$$

In Sec. III A we reproduce (25) and (27) from the dispersion relation of the hydrodynamic QNMs.

Note that the "disordered phase" corresponds to identical vanishing of the bulk scalar field, in this case

$\chi_{4}=c_{0}^{h}=0, \quad a_{1,0}^{h}=1 \Rightarrow \kappa_{\text {disordered }}=1, \quad\langle\hat{\mathcal{O}}\rangle=0$.

\footnotetext{
${ }^{8}$ Eling-Oz formula implies that the holographic plasma bulk viscosity is proportional to the square of the derivative of the bulk scalar field(s) evaluated at the horizon with respect to the temperature, keeping all the mass parameters fixed $[13,14]$.
} 

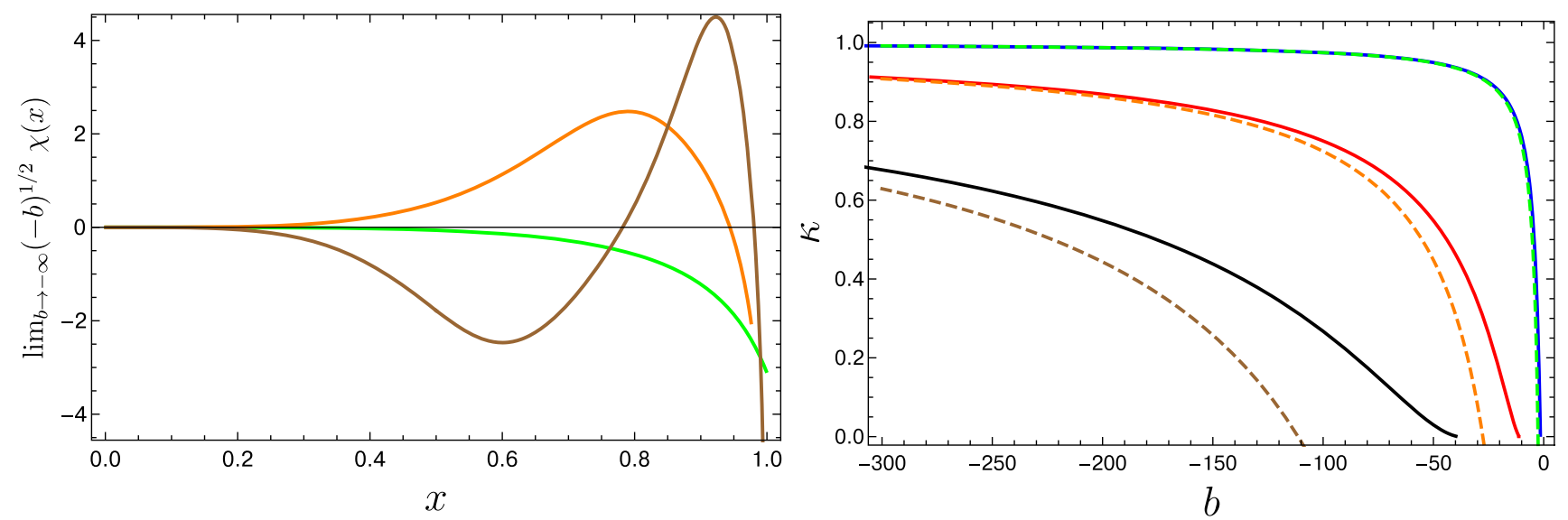

FIG. 1. Left: branches ("overtones") of the holographic conformal order are characterized by the number of zeros of the gravitational scalar dual to the order parameter. We plot the scalar profiles as $b \rightarrow-\infty$. The green/orange/brown profiles correspond to an index $i=0,1,2$ branch/overtone. Right: $\kappa(b)$ characterizing the thermodynamics of the ordered phases [see Eq. (1)] for $i=0,1,2$ (solid blue/ red/black curves correspondingly). The dashed green/orange/brown curves represent the leading order $b \rightarrow-\infty$ approximation, see Eq. (35).

To understand the ordered phases it is the easiest to consider the $b \rightarrow-\infty$ limit. From (18)-(20) it is straightforward to see that

$$
\begin{aligned}
\chi(x) & =\sum_{n=1}^{\infty} \frac{f_{[2 n-1]}(x)}{(-b)^{n-1 / 2}}, \quad a_{1}(x)=1+\sum_{n=1}^{\infty} \frac{a_{1,[n]}(x)}{(-b)^{n}}, \\
a_{3}(x) & =1+\sum_{n=1}^{\infty} \frac{a_{3,[n]}(x)}{(-b)^{n}},
\end{aligned}
$$

and correspondingly [from (21) $a_{1,3} \equiv-a_{3,3}$ ]

$$
\begin{aligned}
a_{3,3} & =\sum_{n=1}^{\infty} \frac{a_{3,[n], 3}}{(-b)^{n}}, \quad \chi_{4}=\sum_{n=1}^{\infty} \frac{f_{[2 n-1], 4}}{(-b)^{n-1 / 2}}, \\
c_{0}^{h} & =\sum_{n=1}^{\infty} \frac{f_{[2 n-1], 0}^{h}}{(-b)^{n-1 / 2}}, \quad a_{1,0}^{h}=1+\sum_{n=1}^{\infty} \frac{a_{1,[n], 0}^{h}}{(-b)^{n}},
\end{aligned}
$$

i.e., in this limit the "hairy" black branes approach the standard $\mathrm{AdS}_{4}$-Schwarzschild solution. ${ }^{9}$ To leading order, i.e., $n=1$, we have

$$
\begin{array}{r}
0=f_{[1]}^{\prime \prime}+\frac{x^{3}+2}{x\left(x^{3}-1\right)} f_{[1]}^{\prime}-\frac{4\left(f_{[1]}^{2}-1\right) f_{[1]}}{x^{2}\left(x^{3}-1\right)}, \\
0=a_{3,[1]}^{\prime}+\frac{3 a_{3,[1]}}{x\left(x^{3}-1\right)}+\frac{x}{8}\left(f_{[1]}^{\prime}\right)^{2}+\frac{f_{[1]}^{2}\left(f_{[1]}^{2}-2\right)}{4 x\left(x^{3}-1\right)},
\end{array}
$$

\footnotetext{
${ }^{9}$ As we will see in Sec. III B, this is not the case for the spectrum of the nonhydrodynamic QNMs: some of the QNMs of the hairy black branes remain distinct from the $\mathrm{AdS}_{4}$-Schwarzschild black brane QNMs in the limit $b \rightarrow-\infty$.
}

$$
0=a_{1,[1]}^{\prime}-\frac{3 a_{3,[1]}}{x\left(x^{3}-1\right)}+\frac{x}{8}\left(f_{[1]}^{\prime}\right)^{2}-\frac{f_{[1]}^{2}\left(f_{[1]}^{2}-2\right)}{4 x\left(x^{3}-1\right)} .
$$

There is a discrete set of solutions of (31) subject to the boundary conditions inherited from (21) and (22), characterized by the number of zeroes in the function $f_{[1]}$. In the left panel of Fig. 1 we present

$$
f_{[1]}(x) \equiv \lim _{b \rightarrow-\infty}(-b)^{1 / 2} \chi(x),
$$

with $i=0,1,2$ (green, orange, brown curves correspondingly) zeros. The discrete set of solutions for (31) leads to discrete sets of $a_{3,[1]}$ and $a_{1,[1]}$. We just constructed the lowest ${ }^{10}(i=0)$ and the higher $(i=1,2)$ overtones of the holographic conformal order:

\begin{tabular}{lcccc} 
Number of zeroes & $f_{1,[4]}$ & $f_{[1], 0}^{h}$ & $a_{3,[1], 3}$ & $a_{1,[1], 0}^{h}$ \\
\hline 0 & \pm 0.914 & \pm 3.114 & 0.644 & -4.928 \\
1 & \pm 7.875 & $\mp 6.789$ & 6.899 & -155.534 \\
2 & \pm 30.546 & \pm 11.233 & 27.835 & -1249.88
\end{tabular}

where \pm for the parameters specifying $f_{[1]}$ reflects the spontaneously broken global $\mathbb{Z}_{2}$ symmetry. To leading order as $b \rightarrow-\infty$ [see (24)]

$\kappa=1+\frac{\left.2 a_{1,[1], 0]}^{h}+\frac{1}{6}\left(f_{[1], 0}^{h}\right)^{4}-\frac{1}{3}\left(f_{[1], 0}^{h}\right)^{2}\right)}{b}+\mathcal{O}\left(\frac{1}{b^{2}}\right)$,

which is represented by the dashed green/orange/brown (for $i=0,1,2$ correspondingly) curves in the right panel of

\footnotetext{
${ }^{10}$ Only this overtone was discussed in [2].
} 

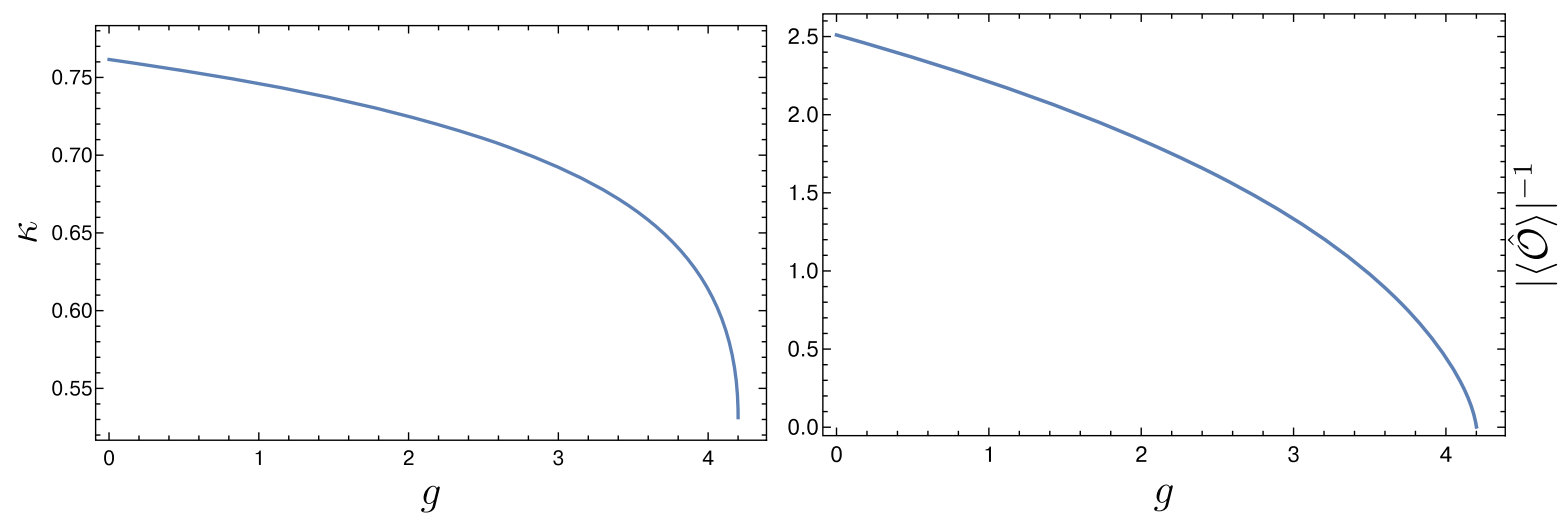

FIG. 2. Left: $\kappa$ parameter of the lowest branch of the conformal order at $b=-10$ as a function of $g$, a deformation parameter bounding the potential of the bulk scalar dual to the order parameter, see Eq. (7). Holographic conformal order persists for $0 \leq g<g_{\text {crit }} \approx 4.2$. Right: the corresponding dependence of the thermal order parameter $\langle\hat{\mathcal{O}}\rangle$. Note that the order parameter diverges as $g \rightarrow g_{\text {crtit }}$.

Fig. 1. Once the overtones of the conformal order are constructed in the limit $b \rightarrow-\infty$, it is straightforward to solve Eqs. (18)-(20) and extend the results for $\kappa_{i}$ to finite values of $b$. This is shown with the solid blue/red/black (for $i=0,1,2$ correspondingly) curves in the right panel. For each overtone of the conformal order there is a critical value of $b$, i.e., $b_{\text {crit }, i}$, beyond which the overtone disappears from the spectrum $^{11}$ :

\begin{tabular}{lccc}
\hline$i=$ number of zeroes & 0 & 1 & 2 \\
\hline$b_{\text {crit }, i}$ & -1.5 & -11.258 & -39.295
\end{tabular}

Note that

$$
\lim _{b \rightarrow b_{\text {crit }, i}} \kappa_{i}(b)=0
$$

and for each $i<j$,

$$
1>\kappa_{i}(b)>\kappa_{j}(b) \quad \text { and } \quad b_{\text {crit }, i}<b_{\text {crit }, j} .
$$

Thus, all the ordered phases are subdominant (have the higher free energy density) compared to the $\mathbb{Z}_{2}$-symmetric phase, see (28). Additionally, the free energy density of the conformal order overtones increases (at fixed $b$ ) with its index.

Equilibrium thermal phases with or without the global $\mathbb{Z}_{2}$ symmetry have positive specific heat. There is a latent heat $\Delta \mathcal{E}$ associated with the transitions between the symmetry broken phases $i<j$, and the transitions to the $\mathbb{Z}_{2}$ symmetric phase,

$$
\Delta \mathcal{E}_{j \rightarrow i} \propto+\left(\kappa_{i}-\kappa_{j}\right) T^{3}, \quad \Delta \mathcal{E}_{i \rightarrow \text { disordered }} \propto+\left(1-\kappa_{i}\right) T^{3},
$$

\footnotetext{
${ }^{11}$ In all cases as $b \rightarrow b_{\text {crit }, i}$ the order parameter $\langle\hat{\mathcal{O}}\rangle_{i}$ diverges, see also [2].
}

typically indicative of the first-order phase transition. Rather, as we show in Sec. III B, each of the ordered phases suffers the perturbative instability for any value of $b$ it exists. A natural guess is that the end point of the dynamical evolution will bring us from the ordered phase to a disordered, $\mathbb{Z}_{2}$-symmetric, phases. However, to confirm this, a numerical simulation is necessary. ${ }^{12}$

\section{B. Holographic conformal order with the bounded gravitational potential}

The gravitational potential for a scalar field dual to a conformal order parameter is unbounded from below; see (6) and note that the existence of the conformal order requires $b<-\frac{3}{2}$. We used simple scaling arguments in Sec. I to suggest that the conformal order exists once the scalar potential is made bounded as in (7), at least for sufficiently small $g$ and in the limit $b \rightarrow-\infty$.

What happens at finite $b$ ? First, note that all the analyses in Sec. II allow for a simple generalization as $V$ is replaced with $V_{g}$ of (7)—see Eqs. (18)-(20) and (24). In the left panel of Fig. 2 we show the results for the parameter $\kappa$ characterizing the thermodynamics of the lowest branch of the conformal order [see (1)] evaluated at $b=-10$ as a function of $g>0$. We find that the holographic conformal order persists for

$$
g \in\left[0, g_{\text {crit }}\right),\left.\quad g_{\text {crit }}\right|_{b=-10} \approx 4.2 .
$$

As $g \rightarrow g_{\text {crit }}$, the order parameter $|\langle\hat{\mathcal{O}}\rangle|$ diverges, see the right panel of Fig. 2.

It is clear that the conformal order constructed in Sec. II is robust against the deformation of the bulk scalar potential as in (7) for $0 \leq g<g_{\text {crit }}(b)$ since the basic equations determining it, i.e., Eqs. (18)-(20) and (24), are analytic in the limit $g \rightarrow 0_{+}$.

\footnotetext{
${ }^{12}$ Another possibility is the evolution to a naked singularity in a dual gravitational description, see $[16,17]$.
} 


\section{QNMS OF THE CONFORMAL ORDER}

In this section we study the spectrum of the linearized fluctuations about hairy black branes, dual to the conformal order-the spectrum of the quasinormal modes [5]:

(i) In Sec. III A we consider the hydrodynamic modes, i.e., QNMs such that $\mathfrak{w}(\mathfrak{q}) \rightarrow$ as $\mathfrak{q} \rightarrow 0$. We confirm the conformal speed of the sound waves (25), the vanishing of the bulk viscosity (27), and the universality of the ratio of the shear viscosity to the entropy density [6-8].

(ii) In Sec. III B we consider the spectrum of the nonhydrodynamic modes. We discuss different branches of the spectra and exhibit the QNM with $\operatorname{Im}[\mathfrak{w}]>0$, for $\mathfrak{q} \leq \mathfrak{q}_{c}$, see Eq. (12). This mode makes holographic conformal order of [2] perturbatively unstable. Its existence is yet another counterexample of the correlated stability conjecture $[9,10]$.

For the most part we focus on the QNM spectra of the lowest branch of the conformal order with a dual gravitational action (2). We discuss however the instability of the higher overtones of the conformal order.

Consider fluctuations of the background geometry (14)

$$
g_{\mu \nu} \rightarrow g_{\mu \nu}+h_{\mu \nu}, \quad \chi \rightarrow \chi+f .
$$

For convenience, we partially fix the gauge by requiring

$$
h_{t r}=h_{x_{i} r}=h_{r r}=0 .
$$

We orient the coordinate system in such a way that the $x_{2}$ axis is directed along the spatial momentum, and assume that all the fluctuations depend only on $\left(t, x_{2}, r\right)$, i.e., we have a $\mathbb{Z}_{2}$ parity symmetry along the $x_{1}$ axis. At a linearized level, the following sets of fluctuations decouple from each other

$$
\begin{aligned}
\mathbb{Z}_{2} \text { - even: } & \left\{h_{t t}, h_{t x_{2}}, h_{x_{1} x_{1}}, h_{x_{2} x_{2}} ; f\right\} ; \\
\mathbb{Z}_{2} \text { - odd }: & \left\{h_{t x_{1}}, h_{x_{1} x_{2}}\right\} .
\end{aligned}
$$

Let

$$
\begin{aligned}
& h_{t t}=-e^{-i \omega t+i k x_{2}} c_{1}(r)^{2} H_{t t}, \quad h_{x_{i} x_{i}}=e^{-i \omega t+i k x_{2}} c_{2}(r)^{2} H_{x_{i} x_{i}}, \\
& h_{t x_{i}}=e^{-i \omega t+i k x_{2}} c_{2}(r)^{2} H_{t x_{i}}, \quad h_{x_{1} x_{2}}=e^{-i \omega t+i k x_{2}} c_{2}(r)^{2} H_{x_{1} x_{2}} \text {, } \\
& f=e^{-i \omega t+i k x_{2}} F,
\end{aligned}
$$

where $\left\{H_{t t}, H_{t x_{i}}, H_{x_{i} x_{i}}, H_{x_{1} x_{2}}, F\right\}$ are functions of the radial coordinate only and $c_{i}(r)$ are defined in (17). Following $[5,18]$, we introduce fluctuations invariant under the residual diffeomorphisms preserving (41):

(i) $\mathbb{Z}_{2}$-even, the sound channel,

$$
\begin{aligned}
Z_{H} & \equiv 4 \frac{k}{\omega} H_{t x_{2}}+2 H_{x_{2} x_{2}}-2 H_{x_{1} x_{1}}\left(1-\frac{k^{2}}{\omega^{2}} \frac{\left(c_{1}^{2}\right)^{\prime}}{\left(c_{2}^{2}\right)^{\prime}}\right)-2 \frac{k^{2}}{\omega^{2}} \frac{c_{1}^{2}}{c_{2}^{2}} H_{t t}, \\
Z_{F} & \equiv f-\frac{\chi^{\prime}}{\left(\ln c_{2}^{2}\right)^{\prime}} H_{x_{1} x_{1}}
\end{aligned}
$$

(ii) $\mathbb{Z}_{2}$-odd, the shear channel,

$$
Z_{s} \equiv k H_{t x_{1}}+\omega H_{x_{1} x_{2}} .
$$

Using the radial coordinate (16), we find the following equations of motion: for the sound channel,

$$
\begin{gathered}
0=Z_{H}^{\prime \prime}+\mathcal{A}_{H} Z_{H}^{\prime}+\mathcal{B}_{H} Z_{H}+\mathcal{C}_{H} Z_{F}, \\
0=Z_{F}^{\prime \prime}+\mathcal{A}_{F} Z_{F}^{\prime}+\mathcal{B}_{F} Z_{H}^{\prime}+\mathcal{C}_{F} Z_{F}+\mathcal{D}_{F} Z_{H},
\end{gathered}
$$

with

$$
\begin{aligned}
\mathcal{A}_{H}= & \left(a_{1}^{2}(\alpha \mathfrak{q})^{2} x^{4}\left(x^{3}-1\right)^{2}\left(\chi^{\prime}\right)^{4}+a_{1}^{2}(\alpha \mathfrak{q})^{2} x^{2}\left(x^{3}-1\right)\left(a_{3}^{2} \tilde{V}+6\left(1-x^{3}\right)\right)\left(\chi^{\prime}\right)^{2}\right. \\
& -2 a_{3}^{4} a_{1}^{2}(\alpha \mathfrak{q})^{2} \tilde{V}^{2}+8 a_{3}^{2}\left(a_{1}^{2}(\alpha \mathfrak{q})^{2}\left(x^{3}-1\right)-2(\alpha \mathfrak{w})^{2}\right) \tilde{V}-8\left(x^{3}-1\right)\left(5 a_{1}^{2}(\alpha \mathfrak{q})^{2}\left(x^{3}-1\right)\right. \\
& \left.\left.-4(\alpha \mathfrak{w})^{2}\right)\right)\left(2 x ( x ^ { 3 } - 1 ) \left(a_{1}^{2}(\alpha \mathfrak{q})^{2} x^{2}\left(x^{3}-1\right)\left(\chi^{\prime}\right)^{2}+2 a_{3}^{2} a_{1}^{2}(\alpha \mathfrak{q})^{2} \tilde{V}+4\left(a_{1}^{2}(\alpha \mathfrak{q})^{2}\left(x^{3}-1\right)\right.\right.\right. \\
& \left.\left.\left.+4(\alpha \mathfrak{w})^{2}\right)\right)\right)^{-1},
\end{aligned}
$$




$$
\begin{aligned}
& \mathcal{B}_{H}=(\alpha \mathfrak{w})^{2}\left(a_{1}^{4}(\alpha \mathfrak{q})^{2} x^{6}\left(x^{3}-1\right)^{3}\left(\chi^{\prime}\right)^{6}+4 a_{1}^{4}(\alpha \mathfrak{q})^{2} x^{4}\left(x^{3}-1\right)^{2}\left(a_{3}^{2} \tilde{V}+5\left(1-x^{3}\right)\right)\left(\chi^{\prime}\right)^{4}\right. \\
& +4 a_{1}^{2}(\alpha \mathfrak{q})^{2} x^{2}\left(x^{3}-1\right)\left(a_{3}^{4} a_{1}^{2} \tilde{V}^{2}-8 a_{3}^{2} a_{1}^{2}\left(x^{3}-1\right) \tilde{V}+4 a_{3}^{2}(\alpha \mathfrak{w})^{2} x^{2}+4 a_{1}^{2}\left(x^{3}-1\right)\left(a_{3}^{2}(\alpha \mathfrak{q})^{2}\right.\right. \\
& \left.\left.\times x^{2}+3\left(x^{3}-1\right)\right)\right)\left(\chi^{\prime}\right)^{2}+16 a_{3}^{4} a_{1}^{4}(\alpha \mathfrak{q})^{2}\left(x^{3}-1\right) \tilde{V}^{2}+32 a_{3}^{2} a_{1}^{2}(\alpha \mathfrak{q})^{2}\left(a _ { 1 } ^ { 2 } ( x ^ { 3 } - 1 ) \left(a_{3}^{2}(\alpha \mathfrak{q})^{2} x^{2}\right.\right. \\
& \left.\left.+6\left(1-x^{3}\right)\right)+a_{3}^{2}(\alpha \mathfrak{w})^{2} x^{2}\right) \tilde{V}+64 a_{1}^{4}(\alpha \mathfrak{q})^{2}\left(x^{3}-1\right)^{2}\left(a_{3}^{2}(\alpha \mathfrak{q})^{2} x^{2}+9\left(x^{3}-1\right)\right) \\
& \left.+320 a_{1}^{2}(\alpha \mathfrak{q})^{2} x^{2} a_{3}^{2}\left(x^{3}-1\right)(\alpha \mathfrak{w})^{2}+256 a_{3}^{2}(\alpha \mathfrak{w})^{4} x^{2}\right)\left(1 6 a _ { 1 } ^ { 2 } ( x ^ { 3 } - 1 ) ^ { 2 } \left(a_{1}^{2}(\alpha \mathfrak{q})^{2}(\alpha \mathfrak{w})^{2} x^{4}\right.\right. \\
& \left.\left.\times\left(x^{3}-1\right)\left(\chi^{\prime}\right)^{2}+2 x^{2}(\alpha \mathfrak{w})^{2}(\alpha \mathfrak{q})^{2} a_{1}^{2} a_{3}^{2} \tilde{V}+4 x^{2}(\alpha \mathfrak{w})^{2}\left(a_{1}^{2}(\alpha \mathfrak{q})^{2}\left(x^{3}-1\right)+4(\alpha \mathfrak{w})^{2}\right)\right)\right)^{-1}, \\
& \mathcal{C}_{H}=a_{1}^{2}(\alpha \mathfrak{q})^{2}\left(\left(\chi^{\prime}\right)^{2} x^{2}\left(x^{3}-1\right)+2 a_{3}^{2} \tilde{V}+12\left(1-x^{3}\right)\right)\left((\alpha \mathfrak{w})^{2} x^{3}\left(x^{3}-1\right)\left(\chi^{\prime}\right)^{3}\right. \\
& +\frac{1}{4} a_{1}^{2}(\alpha \mathfrak{q})^{2} x^{2} a_{3}^{2}\left(x^{3}-1\right) \partial \tilde{V}\left(\chi^{\prime}\right)^{2}-2\left(x^{3}-1\right)\left(6 x(\alpha \mathfrak{w})^{2}+a_{3}^{2} a_{1}^{2}(\alpha \mathfrak{q})^{2} x \tilde{V}\right) \chi^{\prime}+a_{3}^{2}\left(a_{1}^{2}(\alpha \mathfrak{q})^{2}\right. \\
& \left.\left.\times\left(x^{3}-1\right)+4(\alpha \mathfrak{w})^{2}\right) \partial \tilde{V}+\frac{1}{2} a_{3}^{4} a_{1}^{2}(\alpha \mathfrak{q})^{2} \tilde{V} \partial \tilde{V}\right)\left(( x ^ { 3 } - 1 ) x ^ { 2 } ( \alpha \mathfrak { w } ) ^ { 2 } \left(a_{1}^{2}(\alpha \mathfrak{q})^{2} x^{2}\left(x^{3}-1\right)\right.\right. \\
& \left.\left.\times\left(\chi^{\prime}\right)^{2}+2 a_{3}^{2} a_{1}^{2}(\alpha \mathfrak{q})^{2} \tilde{V}+4 a_{1}^{2}(\alpha \mathfrak{q})^{2}\left(x^{3}-1\right)+16(\alpha \mathfrak{w})^{2}\right)\right)^{-1}, \\
& \mathcal{A}_{F}=-\frac{a_{3}^{2} \tilde{V}+2\left(1-x^{3}\right)}{2\left(x^{3}-1\right) x}, \\
& \mathcal{B}_{F}=2 a_{3}^{2}(\alpha \mathfrak{w})^{2}\left(\chi^{\prime} x \tilde{V}-2 \partial \tilde{V}\right)\left(( x ^ { 3 } - 1 ) x \left(a_{1}^{2}(\alpha \mathfrak{q})^{2} x^{2}\left(x^{3}-1\right)\left(\chi^{\prime}\right)^{2}+2 a_{3}^{2} a_{1}^{2}(\alpha \mathfrak{q})^{2} \tilde{V}\right.\right. \\
& \left.\left.+4 a_{1}^{2}(\alpha \mathfrak{q})^{2}\left(x^{3}-1\right)+16(\alpha \mathfrak{w})^{2}\right)\right)^{-1}, \\
& \mathcal{C}_{F}=-a_{3}^{2}\left(\frac{1}{2} a_{1}^{4}(\alpha \mathfrak{q})^{2} x^{3}\left(x^{3}-1\right)^{2} \partial \tilde{V}\left(\chi^{\prime}\right)^{3}+\left(-4 a_{1}^{2} x^{2}\left(x^{3}-1\right)\left(a_{1}^{2}(\alpha \mathfrak{q})^{2}\left(x^{3}-1\right)+(\alpha \mathfrak{w})^{2}\right)\right.\right. \\
& \times \tilde{V}-a_{1}^{4}(\alpha \boldsymbol{q})^{2} x^{2}\left(x^{3}-1\right)^{2} \partial^{2} \tilde{V}-a_{1}^{2}(\alpha \mathfrak{q})^{2} x^{2}\left(x^{3}-1\right)\left(a_{1}^{2}\left(x^{3}-1\right)\left((\alpha \mathfrak{q})^{2} x^{2}+4\right)\right. \\
& \left.\left.+(\alpha \mathfrak{w})^{2} x^{2}\right)\right)\left(\chi^{\prime}\right)^{2}+a_{1}^{2} x\left(x^{3}-1\right) \partial \tilde{V}\left(a_{3}^{2} a_{1}^{2}(\alpha \mathfrak{q})^{2} \tilde{V}+10 a_{1}^{2}(\alpha \mathfrak{q})^{2}\left(x^{3}-1\right)+16(\alpha \mathfrak{w})^{2}\right) \chi^{\prime} \\
& -2\left(a_{1}^{2}\left(x^{3}-1\right) \partial^{2} \tilde{V}+a_{1}^{2}\left(x^{3}-1\right)\left((\alpha \mathfrak{q})^{2} x^{2}+4\right)+(\alpha \mathfrak{w})^{2} x^{2}\right)\left(a_{3}^{2} a_{1}^{2}(\alpha \mathfrak{q})^{2} \tilde{V}+2 a_{1}^{2}(\alpha \mathfrak{q})^{2}\right. \\
& \left.\left.\times\left(x^{3}-1\right)+8(\alpha \mathfrak{w})^{2}\right)\right)\left(( x ^ { 3 } - 1 ) ^ { 2 } a _ { 1 } ^ { 2 } x ^ { 2 } \left(a_{1}^{2}(\alpha \mathfrak{q})^{2} x^{2}\left(x^{3}-1\right)\left(\chi^{\prime}\right)^{2}+2 a_{3}^{2} a_{1}^{2}(\alpha \mathfrak{q})^{2} \tilde{V}+4 a_{1}^{2}\right.\right. \\
& \left.\left.\times(\alpha \mathfrak{q})^{2}\left(x^{3}-1\right)+16(\alpha \mathfrak{w})^{2}\right)\right)^{-1}, \\
& \mathcal{D}_{F}=a_{3}^{2}(\alpha \mathfrak{w})^{2}\left(x \tilde{V} \chi^{\prime}-2 \partial \tilde{V}\right)\left(\left(\chi^{\prime}\right)^{2} x^{2}\left(x^{3}-1\right)+2 a_{3}^{2} \tilde{V}+12\left(1-x^{3}\right)\right)\left(4\left(x^{3}-1\right)^{2} x^{2}\right. \\
& \left.\times\left(a_{1}^{2}(\alpha \mathfrak{q})^{2} x^{2}\left(x^{3}-1\right)\left(\chi^{\prime}\right)^{2}+2 a_{3}^{2} a_{1}^{2}(\alpha \mathfrak{q})^{2} \tilde{V}+4 a_{1}^{2}(\alpha \mathfrak{q})^{2}\left(x^{3}-1\right)+16(\alpha \mathfrak{w})^{2}\right)\right)^{-1},
\end{aligned}
$$

where we introduced

$$
\begin{aligned}
& \tilde{V}=V-6, \quad \partial \tilde{V}=\frac{\delta \tilde{V}}{\delta \chi}, \quad \partial^{2} \tilde{V}=\frac{\delta^{2} \tilde{V}}{\delta \chi^{2}}, \\
& \mathfrak{q}=\frac{k}{4 \pi T}=\frac{k}{r_{0}} \frac{1}{\alpha}, \quad \mathfrak{w}=\frac{\omega}{4 \pi T}=\frac{\omega}{r_{0}} \frac{1}{\alpha}, \quad \alpha=a_{1,0}^{h} \sqrt{9-\frac{3}{2} V\left(c_{0}^{h}\right),}
\end{aligned}
$$

with $V$ being the scalar potential (6); and for the shear channel,

$$
0=Z_{s}^{\prime \prime}+\mathcal{A}_{s} Z_{s}^{\prime}+\mathcal{B}_{s} Z_{s}
$$

with

$$
\begin{aligned}
\mathcal{A}_{s}= & \left(a_{1}^{2}(\alpha \mathfrak{q})^{2} x^{2}\left(x^{3}-1\right)^{2}\left(\chi^{\prime}\right)^{2}-2 a_{3}^{2}(\alpha \mathfrak{w})^{2} \tilde{V}-\left(4\left(x^{3}-1\right)\right)\left(2 a_{1}^{2}(\alpha \mathfrak{q})^{2}\left(x^{3}-1\right)\right.\right. \\
& \left.\left.-(\alpha \mathfrak{w})^{2}\right)\right)\left(4 x\left(x^{3}-1\right)\left(a_{1}^{2}(\alpha \mathfrak{q})^{2}\left(x^{3}-1\right)+(\alpha \mathfrak{w})^{2}\right)\right)^{-1},
\end{aligned}
$$




$$
\mathcal{B}_{s}=\frac{a_{3}^{2}\left(a_{1}^{2}(\alpha \mathfrak{q})^{2}\left(x^{3}-1\right)+(\alpha \mathfrak{w})^{2}\right)}{\left(x^{3}-1\right)^{2} a_{1}^{2}} .
$$

\section{A. Hydrodynamic modes and the transport}

We begin with the $\mathfrak{q} \rightarrow 0$ limit of the dispersion relation for the shear and the sound channels QNM modes. From the former, we extract the shear viscosity, and from the latter, the speed of the sound waves and the bulk viscosity of the holographic conformal order. We discuss the dispersion relation $\mathfrak{w}=\mathfrak{w}(\mathfrak{q})$ for the sound waves in Sec. III A 3 for different values of $b$.

\section{The shear viscosity}

The shear mode dispersion relation in the limit $\mathfrak{q} \rightarrow 0$ takes the form

$$
\mathfrak{w}=-i \frac{4 \pi \eta}{s} \mathfrak{q}^{2}+\mathcal{O}\left(\mathfrak{q}^{4}\right)
$$

We now evaluate (59) in the holographic conformal order. To this end, we set

$$
\begin{aligned}
Z_{s} & =x^{3}(1-x)^{-i \mathfrak{w}}\left(Z_{s, 0}+\mathfrak{q}^{2} Z_{s, 2}+\mathcal{O}\left(\mathfrak{q}^{4}\right)\right) \\
\mathfrak{w} & =-i \beta \mathfrak{q}^{2}+\mathcal{O}\left(\mathfrak{q}^{4}\right)
\end{aligned}
$$

Using (56), we find

$$
\begin{aligned}
& 0=Z_{s, 0}^{\prime \prime}+\frac{x^{2}\left(\chi^{\prime}\right)^{2}+16}{4 x} Z_{s, 0}{ }^{\prime}+\frac{3}{4}\left(\chi^{\prime}\right)^{2} Z_{s, 0}, \\
& 0=Z_{s, 2}^{\prime \prime}+\frac{x^{2}\left(\chi^{\prime}\right)^{2}+16}{4 x} Z_{s, 2}{ }^{\prime}+\frac{3}{4}\left(\chi^{\prime}\right)^{2} Z_{s, 2}+J_{s, 2},
\end{aligned}
$$

where

$$
\begin{aligned}
J_{s, 2}= & \beta\left(\frac{x \beta\left(\chi^{\prime}\right)^{2}}{4 a_{1}^{2}\left(x^{3}-1\right)}+\frac{\beta a_{3}^{2} \tilde{V}}{2 x\left(x^{3}-1\right)^{2} a_{1}^{2}}-\frac{3 \beta}{a_{1}^{2}\left(x^{3}-1\right) x}-\frac{2}{x-1}\right) Z_{s, 0}{ }^{\prime}-\left(\left(\chi^{\prime}\right)^{2}\right. \\
& \left.\times \frac{\beta\left(a_{1}^{2} x\left(x^{2}+x+1\right)-3 \beta\right)}{4\left(x^{3}-1\right) a_{1}^{2}}-\frac{a_{3}^{2} \alpha^{2}}{x^{3}-1}+\frac{(3 x-4) \beta}{x(x-1)^{2}}-\frac{\left(3 a_{3}^{2} \tilde{V}-18\left(x^{3}-1\right)\right) \beta^{2}}{2\left(x^{3}-1\right)^{2} a_{1}^{2} x^{2}}\right) Z_{s, 0} .
\end{aligned}
$$

Equations (61) have to be solved subject to the following boundary conditions:

In the UV, i.e., as $x \rightarrow 0_{+}$,

$$
Z_{s, 0}=1+\mathcal{O}\left(x^{8}\right), \quad Z_{s, 2}=-\beta x+\mathcal{O}\left(x^{2}\right) .
$$

In the IR, i.e., as $y \equiv 1-x \rightarrow 0_{+}$,

$$
Z_{s, 0}=z_{0,0}^{h}+z_{0,1}^{h} y+\mathcal{O}\left(y^{2}\right), \quad Z_{s, 2}=z_{2,0}^{h}+z_{2,1}^{h} y+\mathcal{O}\left(y^{2}\right) .
$$

Given (60), these asymptotes reflect the incoming boundary conditions as the horizon, and the Dirichlet boundary conditions at the asymptotic boundary [5].

Ultimately, we need to extract $\beta=\beta(b)$. First, we need to solve numerically the first equation in (61), producing the datasets $\left\{z_{0,0}^{h}(b), z_{0,1}^{h}(b)\right\}$. Remarkably, we do not need to solve for $Z_{s, 2}$ : direct series expansion of the second equation in (61) in the IR leads to

$$
\begin{aligned}
0= & \frac{\left(3\left(a_{1,0}^{h}\right)^{2} z_{0,0}^{h}-3 \beta z_{0,0}^{h}+\beta z_{0,1}^{h}\right) \beta}{3\left(a_{1,0}^{h}\right)^{2}} \frac{1}{y^{2}}+\frac{1}{y} \frac{3\left(a_{1,0}^{h}\right)^{2} z_{0,0}^{h}-3 \beta z_{0,0}^{h}+\beta z_{0,1}^{h}}{3\left(c_{0}^{h}\right)^{2} \tilde{V}\left(c_{0}^{h}\right)^{2}\left(a_{1,0}^{h}\right)^{2}}\left(16 \beta\left(\left(c_{0}^{h}\right)^{2}-6\right)\left(\left(c_{0}^{h}\right)^{2}-6-2 \tilde{V}\left(c_{0}^{h}\right)\right)\right. \\
& \left.-\left(3\left(a_{1,0}^{h}\right)^{2}\left(c_{0}^{h}\right)^{2}-\beta\left(c_{0}^{h}\right)^{2}-16 \beta\right) \tilde{V}\left(c_{0}^{h}\right)^{2}\right)+\mathcal{O}\left(y^{0}\right),
\end{aligned}
$$

with the parameters of the asymptotic expansion of $Z_{s, 2}$ [see (64)] entering only in $\mathcal{O}(y)$. Thus, we find

$$
\beta=\frac{3\left(a_{1,0}^{h}\right)^{2} z_{0,0}^{h}}{3 z_{0,0}^{h}-z_{0,1}^{h}} .
$$

In the disordered phase, see Eq. (28), the equation for $Z_{s, 0}$ is very simple:

$$
\left.\left.\left.\chi(x)\right|_{\text {disordered }} \equiv 0 \Rightarrow Z_{s, 0}\right|_{\text {disordered }} \equiv 1 \Rightarrow \beta\right|_{\text {disordered }}=1,
$$




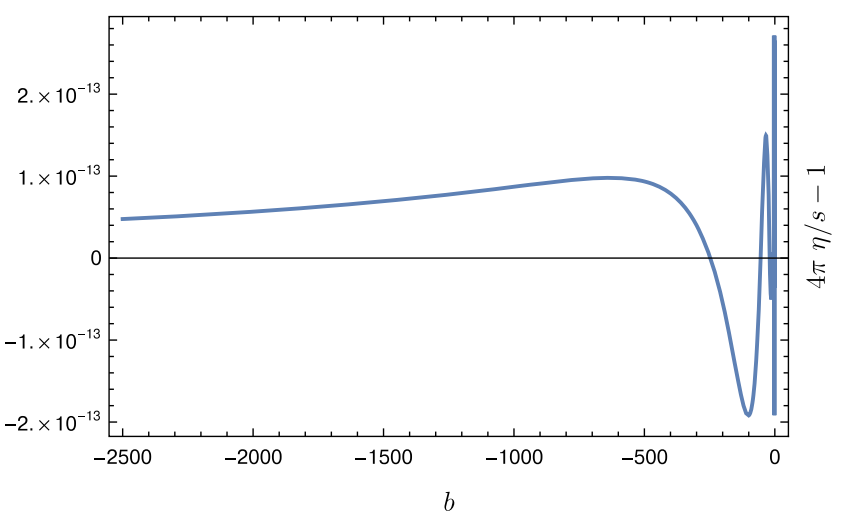

FIG. 3. The ratio of the shear viscosity to the entropy density in the holographic conformal order as a function of $b$ obtained from the analysis of the shear channel QNMs.

leading to the universal result for the ratio of the shear viscosity to the entropy density [6]. In the symmetry broken phase, from the perspective of the shear channel QNM discussed here, we do not have a general proof why $\beta$ must be unity as well. In Fig. 3 we present $\beta-1$ of (66), from the numerical solution of the equation for $Z_{s, 0}$.

We would like to stress that we already know from [6] that ${ }^{13} \beta=1$; the analysis presented here should be viewed as a highly nontrivial check on the QNM equations and our numerical construction of the holographic order parameter.

\section{The speed of the sound waves and the bulk viscosity}

The sound mode dispersion relation in the limit $\mathfrak{q} \rightarrow 0$ takes the form

$$
\mathfrak{w}=c_{s} \mathfrak{q}-\frac{i}{2} \frac{4 \pi \eta}{s}\left(1+\frac{\zeta}{\eta}\right) \mathfrak{q}^{2}+\mathcal{O}\left(\mathfrak{q}^{3}\right) .
$$

We now evaluate (68) in the holographic conformal order. To this end, we set

$$
\begin{aligned}
Z_{H} & =(1-x)^{-i \mathfrak{w}}\left(Z_{H, 0}+i \mathfrak{q} Z_{H, 1}+\mathcal{O}\left(\mathfrak{q}^{2}\right)\right), \\
Z_{F} & =(1-x)^{-i \mathfrak{w}}\left(Z_{F, 0}+i \mathfrak{q} Z_{F, 1}+\mathcal{O}\left(\mathfrak{q}^{2}\right)\right), \\
\mathfrak{w} & =\frac{v}{\sqrt{2}} \mathfrak{q}-\frac{i}{2} \Gamma \mathfrak{q}^{2}+\mathcal{O}\left(\mathfrak{q}^{3}\right) .
\end{aligned}
$$

It is straightforward to derive the corresponding equations of motion from (46) and (47) - they are too long to be presented here. We explain the boundary conditions only:

In the UV, i.e., as $x \rightarrow 0_{+}$,

$$
\begin{aligned}
& Z_{H, 0}=x^{3}+\mathcal{O}\left(x^{6}\right), \quad Z_{F, 0}=z_{f, 0,0} x^{4}+\mathcal{O}\left(x^{7}\right), \\
& Z_{H, 1}=-\frac{v}{\sqrt{2}} x^{4}+\mathcal{O}\left(x^{5}\right), \quad Z_{F, 1}=z_{f, 1,0} x^{4}+\mathcal{O}\left(x^{5}\right) .
\end{aligned}
$$

\footnotetext{
${ }^{13}$ The universality of the shear viscosity in the holographic plasma was never proven from the perspective of the shear channel QNMs dispersion relation.
}

In the IR, i.e., as $y \equiv 1-x \rightarrow 0_{+}$,

$$
\begin{array}{ll}
Z_{H, 0}=z_{h, 0,0}^{h}+\mathcal{O}(y), & Z_{F, 0}=z_{f, 0,0}^{h}+\mathcal{O}(y), \\
Z_{H, 1}=z_{h, 1,0}^{h}+\mathcal{O}(y), & Z_{F, 1}=z_{f, 1,0}^{h}+\mathcal{O}(y) .
\end{array}
$$

Given (69), these asymptotes reflect the incoming boundary conditions as the horizon, and the Dirichlet boundary conditions at the asymptotic boundary [5].

Numerically solving the equations for $\left\{Z_{H, 0}, Z_{F, 0}\right.$, $\left.Z_{H, 1}, Z_{F, 1}\right\}$, subject to the boundary conditions (70) and (71), we extract

$$
1-2 c_{s}^{2}=1-v^{2} \quad \text { and } \quad \frac{\zeta}{\eta}=\Gamma-1
$$

where we used the universal result for the ratio of the shear viscosity to the entropy density [6]. These results are presented in Fig. 4. As expected, the transport in the ordered phase is conformal.

\section{Dispersion of the sound waves in holographic conformal order}

We now present results for the dispersion relation of the sound waves at finite $\mathfrak{q}$ in holographic conformal order. We set

$$
\begin{aligned}
\mathfrak{w}(\mathfrak{q}) & =\mathfrak{w}_{r}(\mathfrak{q})+i \mathfrak{w}_{i}(\mathfrak{q}), \quad \text { with } \quad \lim _{\mathfrak{q} \rightarrow 0} \mathfrak{w}(\mathfrak{q})=0, \\
Z_{H} & =(1-x)^{-i \mathfrak{w}}\left(Z_{H, r}+i Z_{H, i}\right), \\
Z_{F} & =(1-x)^{-i \mathfrak{w}}\left(Z_{F, r}+i Z_{F, i}\right),
\end{aligned}
$$

and obtain from (46) and (47) equations ${ }^{14}$ for $\left\{Z_{H, r}, Z_{H, i}, Z_{F, r}, Z_{F, i}\right\}$. These equations have to be solved subject to the boundary conditions:

In the UV, i.e., as $x \rightarrow 0_{+}$,

$$
\begin{aligned}
& Z_{H, r}=x^{3}+\mathcal{O}\left(x^{4}\right), \quad Z_{H, i}=-\mathfrak{w}_{r} x^{4}+\mathcal{O}\left(x^{5}\right), \\
& Z_{F, r}=z_{f, r, 0} x^{4}+\mathcal{O}\left(x^{5}\right), \quad Z_{F, i}=z_{f, i, 0} x^{4}+\mathcal{O}\left(x^{5}\right) .
\end{aligned}
$$

In the IR, i.e., as $y \equiv 1-x \rightarrow 0_{+}$,

$$
\begin{aligned}
Z_{H, r} & =z_{h, r, 0}^{h}+\mathcal{O}(y), & & Z_{H, i}=z_{h, i, 0}^{h}+\mathcal{O}(y), \\
Z_{F, r} & =z_{f, r, 0}^{h}+\mathcal{O}(y), & Z_{F, i} & =z_{f, i, 0}^{h}+\mathcal{O}(y) .
\end{aligned}
$$

Given (73), these asymptotes reflect the incoming boundary conditions as the horizon and the Dirichlet boundary conditions at the asymptotic boundary [5].

In Figs. 5 and 6 we present results for $\mathfrak{w}_{r} \equiv \operatorname{Re}[\mathfrak{w}]$ and $\mathfrak{w}_{i} \equiv \operatorname{Im}[\mathfrak{w}]$ of the sound mode in holographic conformal

\footnotetext{
${ }^{14}$ The equations are too long to be presented here.
} 

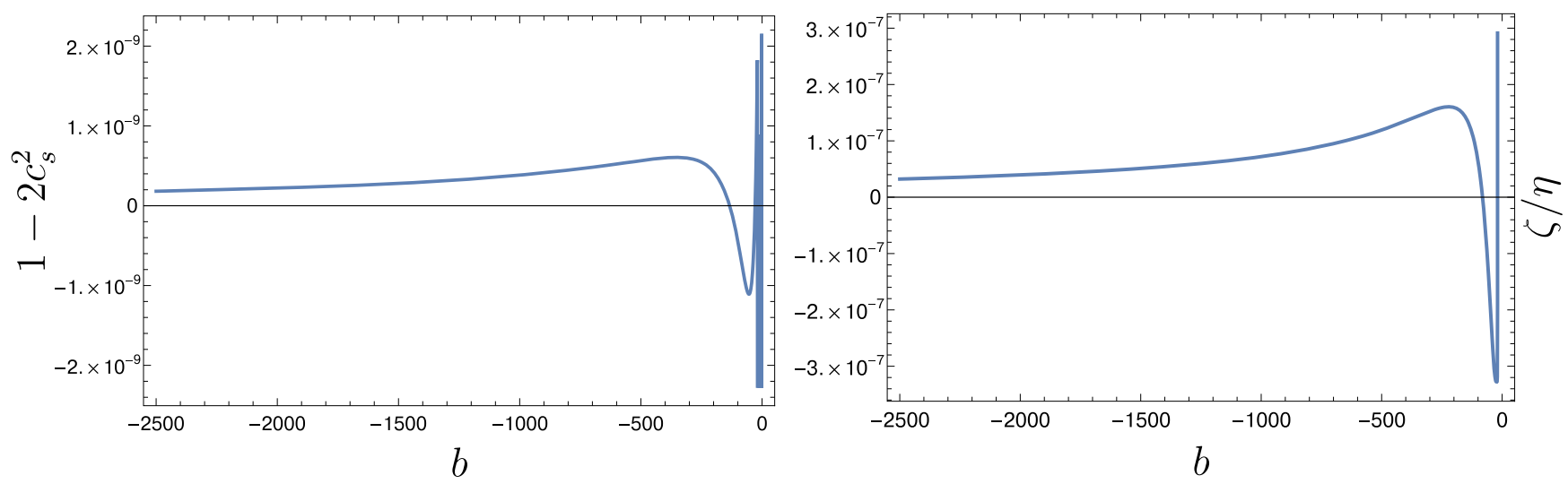

FIG. 4. The speed of the sound waves (left) and the bulk viscosity (right) of the holographic conformal order as a function of $b$ obtained from the analysis of the sound channel QNMs.
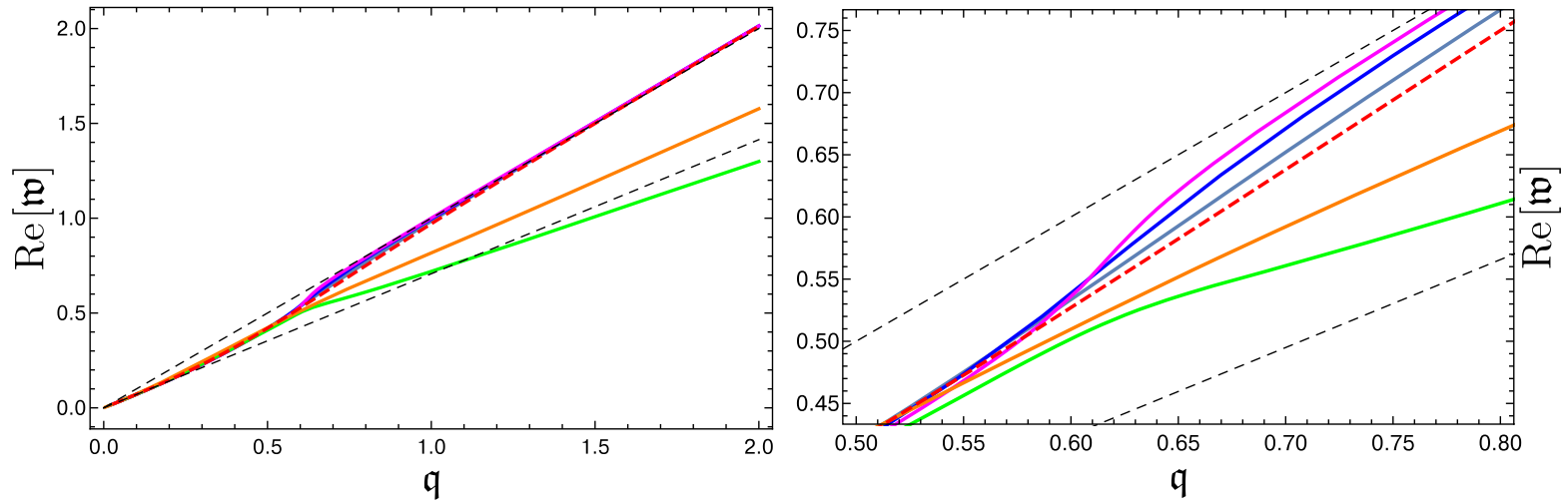

FIG. 5. $\operatorname{Re}[\mathfrak{w}]$ of the sound waves dispersion relation in the disordered (dashed red curve) and ordered phases (solid curves) for select values of $b$; see text. The dashed black lines indicate the hydrodynamic $\mathfrak{q} \rightarrow 0$ and the $\mathfrak{q} \rightarrow \infty$ limits.

order at $b=\{-10,-5,-4,-3,-2\}$ [solid (grey, blue, magenta, green, orange) curves correspondingly]. The dashed red curve represents the dispersion relation for the disordered phase, also in the limit $b \rightarrow-\infty$; see Sec. II A. The dashed black lines represent the hydrodynamic approximation

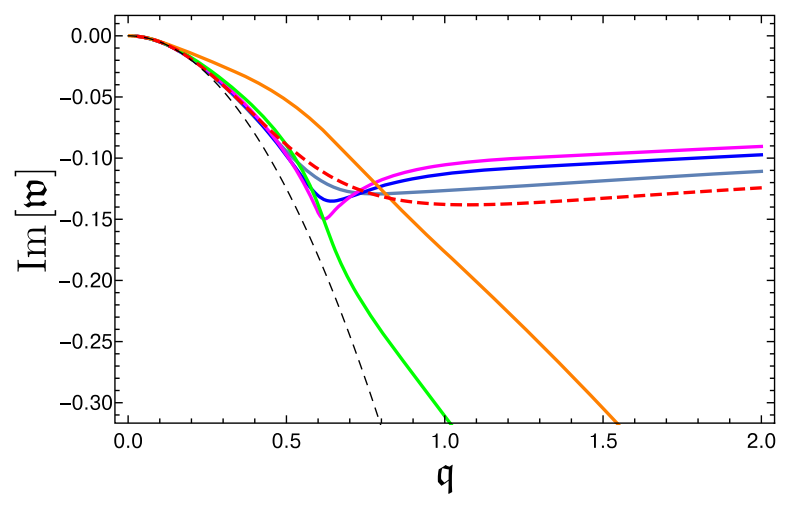

$$
\lim _{\mathfrak{q} \rightarrow 0} \frac{\mathfrak{w}_{r}(\mathfrak{q})}{\mathfrak{q}}=\frac{1}{\sqrt{2}}, \quad \lim _{\mathfrak{q} \rightarrow 0} \frac{\mathfrak{w}_{i}(\mathfrak{q})}{\mathfrak{q}^{2}}=-\frac{1}{2}
$$

and the large $\mathfrak{q}$ limit,

$$
\lim _{\mathfrak{q} \rightarrow \infty} \frac{\mathfrak{w}_{r}(\mathfrak{q})}{\mathfrak{q}}=1 .
$$

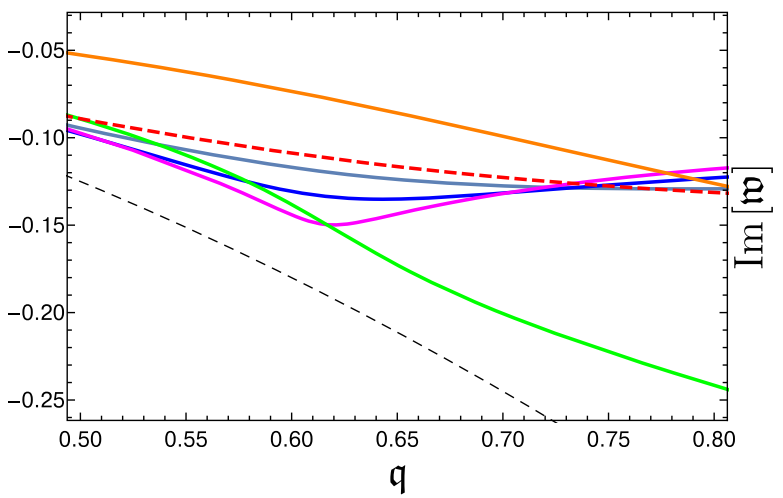

FIG. 6. $\operatorname{Im}[\mathfrak{w}]$ of the sound waves dispersion relation in the disordered (dashed red curve) and ordered phases (solid curves) for select values of $b$; see text. The dashed black line indicates the hydrodynamic $\mathfrak{q} \rightarrow 0$ limit. 
There is a noticeable deviation in the sound QNM mode dispersion between the ordered and the disordered phases for $b \gtrsim-5$; this is a reflection of the fact that in this regime some nonhydrodynamic modes become light; see Fig. 9.

\section{B. Nonhydrodynamic QNMs and the instability}

In this section we discuss the spectrum of low-lying nonhydrodynamic QNMs. We consider only the nonhydrodynamic modes in the sound channel. We identify the QNM with $\operatorname{Im}\left[\mathfrak{w}_{u}\right]>0$, see (12), rendering the holographic conformal order discussed in this paper unstable. This mode is present in the spectrum for all values of $b$, whenever the conformal order is present. We explain why this mode is present in the ordered phases in the limit $^{15} b \rightarrow-\infty$, and is absent in the disordered phase.
To begin, we set $\mathfrak{q}=0$. There are two distinct branches of the sound channel QNMs: we call them $\mathcal{B} \mathcal{R}_{\text {metric }}$ and $\mathcal{B} \mathcal{R}_{\text {hair }}$.

$\mathcal{B} \mathcal{R}_{\text {metric }}$ branch of the sound channel QNMs.-Notice that, see (50),

$$
\left.\mathcal{C}_{H}\right|_{\mathfrak{q}=0}=0
$$

which decouples $Z_{H}$ and $Z_{F}$ fluctuations. On this branch, the spectrum is completely determined by the equation for $Z_{H}$ fluctuations, even though $Z_{F}$ fluctuations are sourced by the former ones: $\mathcal{B}_{F} \neq 0$ and $\mathcal{D}_{F} \neq 0$ in (47). Using the decomposition as in (73), but for $\lim _{\mathfrak{q} \rightarrow 0} \mathfrak{w}(\mathfrak{q}) \neq 0$, we find

$$
\begin{aligned}
& 0=Z_{H, r}^{\prime \prime}+\frac{2\left(x^{2}+x+1\right)\left(2 \mathfrak{w}_{i} x+x-1\right)-a_{3}^{2} \tilde{V}}{2\left(x^{3}-1\right) x} Z_{H, r}^{\prime}+\frac{2 \mathfrak{w}_{r}}{x-1} Z_{H, i}^{\prime}-\frac{1}{2 x a_{1}^{2}\left(x^{3}-1\right)^{2}} \\
& \times\left(2 a_{3}^{2} x\left(\mathfrak{w}_{i}^{2}-\mathfrak{w}_{r}^{2}\right) \alpha^{2}+a_{1}^{2}\left(x^{2}+x+1\right)\left(a_{3}^{2} \tilde{V} \mathfrak{w}_{i}-2\left(x^{2}+x+1\right)\left(\left(\mathfrak{w}_{i}^{2}-\mathfrak{w}_{r}^{2}\right) x-\mathfrak{w}_{i}\right)\right)\right) \\
& \times Z_{H, r}-\frac{\mathfrak{w}_{r}}{2 x a_{1}^{2}\left(x^{3}-1\right)^{2}}\left(a_{3}^{2} a_{1}^{2}\left(x^{2}+x+1\right) \tilde{V}+4 a_{3}^{2} \mathfrak{w}_{i} x \alpha^{2}-2 a_{1}^{2}\left(x^{2}+x+1\right)^{2}\left(2 \mathfrak{w}_{i} x\right.\right. \\
& -1)) Z_{H, i} \text {, } \\
& 0=Z_{H, i}^{\prime \prime}-\frac{a_{3}^{2} \tilde{V}-2\left(x^{2}+x+1\right)\left(2 \mathfrak{w}_{i} x+x-1\right)}{2\left(x^{3}-1\right) x} Z_{H, i}^{\prime}-\frac{2 \mathfrak{w}_{r}}{x-1} Z_{H, r}^{\prime}-\frac{1}{2 x a_{1}^{2}\left(x^{3}-1\right)^{2}} \\
& \times\left(a_{1}^{2} a_{3}^{2} \mathfrak{w}_{i}\left(x^{2}+x+1\right) \tilde{V}+2 a_{3}^{2} x\left(\mathfrak{w}_{i}^{2}-\mathfrak{w}_{r}^{2}\right) \alpha^{2}-2 a_{1}^{2}\left(x^{2}+x+1\right)^{2}\left(\left(\mathfrak{w}_{i}^{2}-\mathfrak{w}_{r}^{2}\right) x-\mathfrak{w}_{i}\right)\right) \\
& \times Z_{H, i}+\frac{\mathfrak{w}_{r}}{2 x a_{1}^{2}\left(x^{3}-1\right)^{2}}\left(a_{1}^{2} a_{3}^{2}\left(x^{2}+x+1\right) \tilde{V}+4 a_{3}^{2} \mathfrak{w}_{i} x \alpha^{2}-2 a_{1}^{2}\left(x^{2}+x+1\right)^{2}\left(2 \mathfrak{w}_{i} x-1\right)\right) Z_{H, r} .
\end{aligned}
$$

The boundary conditions are as in the first lines of Eqs. (74) and (75). Note that the limit $b \rightarrow-\infty$ is trivial here [see (55) and (29)]:

$$
\tilde{V} \rightarrow-6, \quad a_{1} \rightarrow 1, \quad a_{3} \rightarrow 1, \quad \alpha \rightarrow 3,
$$

precisely reproducing the equations for the $\mathfrak{q}=0$ nonhydrodynamic QNMs of the $\mathrm{AdS}_{4}$ Schwarzschild black brane- there is no distinction between the ordered and the disordered QNMs in this limit. We present the spectrum of the quasinormal modes on this branch for $b=-4$ as blue dots in Fig. 7.

$\mathcal{B R}_{\text {hair }}$ branch of the sound channel QNMs.-Setting identically $Z_{H} \equiv 0$, in addition to $\mathfrak{q}=0$, we satisfy the QNM equation (46). Using the decomposition as in (73) we find

\footnotetext{
${ }^{15}$ Recall that in this limit there is no distinction between the ordered and the disordered phases thermodynamics.
}

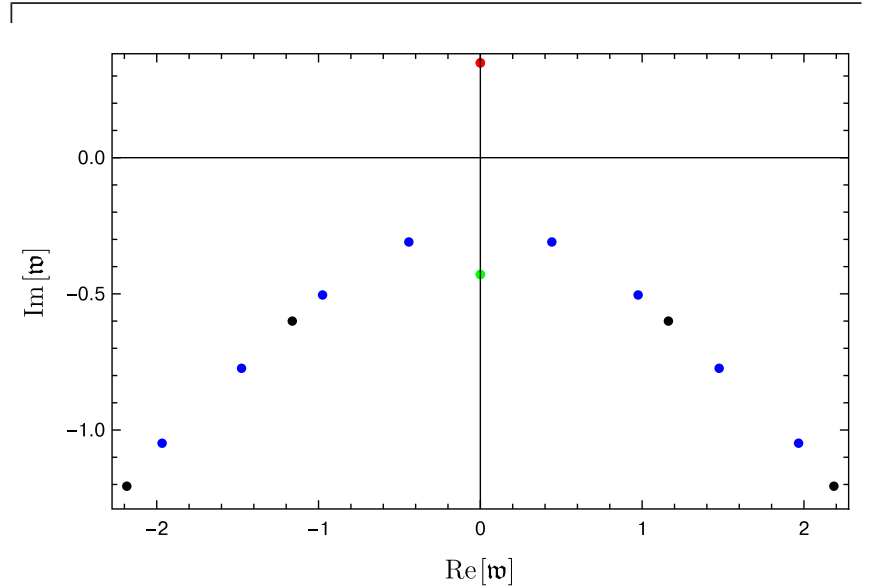

FIG. 7. Spectrum of low-lying QNMs in the ordered phase at $b=-4$. There are two branches $\mathcal{B} \mathcal{R}_{\text {metric }}$ (blue dots) and $\mathcal{B} \mathcal{R}_{\text {hair }}$ (black dots). The QNMs on the former branch reproduce the QNMs of the $\mathrm{AdS}_{4}$-Schwarzschild black brane in the limit $b \rightarrow-\infty$, while those on the $\mathcal{B R}_{\text {hair }}$ branch remain distinct in the limit. QNMs on the $\mathcal{B} \mathcal{R}_{\text {hair }}^{\operatorname{Im}}$ sub-branch of the $\mathcal{B} \mathcal{R}_{\text {hair }}$ branch are nonpropagating: $\operatorname{Re}[\mathfrak{w}]=0$. There is a single dissipative mode (a green dot) and the unstable mode (the red dot). 


$$
\begin{aligned}
0= & Z_{F, r}^{\prime \prime}-\frac{a_{3}^{2} \tilde{V}-2\left(x^{2}+x+1\right)\left(2 \mathfrak{w}_{i} x+x-1\right)}{2\left(x^{3}-1\right) x} Z_{F, r}^{\prime}+\frac{2 \mathfrak{w}_{r}}{x-1} Z_{F, i}^{\prime}+\frac{1}{4 a_{1}^{2} x^{2}\left(x^{3}-1\right)^{2}} \\
& \times\left(4 a_{3}^{2} a_{1}^{2}\left(x^{3}-1\right) \partial^{2} \tilde{V}-4 \chi^{\prime} a_{3}^{2} a_{1}^{2} x\left(x^{3}-1\right) \partial \tilde{V}+a_{3}^{2} a_{1}^{2} x\left(x^{2}+x+1\right)\left(\left(\chi^{\prime}\right)^{2} x(x-1)\right.\right. \\
& \left.-2 \mathfrak{w}_{i}\right) \tilde{V}-4 a_{3}^{2} x^{2}\left(\mathfrak{w}_{i}^{2}-\mathfrak{w}_{r}^{2}\right) \alpha^{2}+4 a_{1}^{2}\left(x^{2}+x+1\right)\left(4 a_{3}^{2}(x-1)+x\left(x^{2}+x+1\right)\right. \\
& \left.\left.\times\left(\left(\mathfrak{w}_{i}^{2}-\mathfrak{w}_{r}^{2}\right) x-\mathfrak{w}_{i}\right)\right)\right) Z_{F, r}-\frac{\mathfrak{w}_{r}}{2 x a_{1}^{2}\left(x^{3}-1\right)^{2}}\left(a_{1}^{2} a_{3}^{2}\left(x^{2}+x+1\right) \tilde{V}+4 a_{3}^{2} \mathfrak{w}_{i} x \alpha^{2}\right. \\
& \left.-2 a_{1}^{2}\left(x^{2}+x+1\right)^{2}\left(2 \mathfrak{w}_{i} x-1\right)\right) Z_{F, i}, \\
0=Z_{F, i}^{\prime \prime} & -\frac{a_{3}^{2} \tilde{V}-2\left(x^{2}+x+1\right)\left(2 \mathfrak{w}_{i} x+x-1\right)}{2 x\left(x^{3}-1\right)} Z_{F, i}^{\prime}-\frac{2 \mathfrak{w}_{r}}{x-1} Z_{F, r}^{\prime}+\frac{1}{4 a_{1}^{2} x^{2}\left(x^{3}-1\right)^{2}} \\
& \times\left(4 a_{3}^{2} a_{1}^{2}\left(x^{3}-1\right) \partial^{2} \tilde{V}-4 \chi^{\prime} a_{3}^{2} a_{1}^{2} x\left(x^{3}-1\right) \partial \tilde{V}-4 a_{3}^{2} x^{2}\left(\mathfrak{w}_{i}^{2}-\mathfrak{w}_{r}^{2}\right) \alpha^{2}+a_{1}^{2}\left(x^{2}+x+1\right)\right. \\
& \left.\times\left(a_{3}^{2} \tilde{V} x\left(\chi^{\prime}\right)^{2} x(x-1)-2 \mathfrak{w}_{i}\right)+16 a_{3}^{2}(x-1)+4 x\left(x^{2}+x+1\right)\left(\left(\mathfrak{w}_{i}^{2}-\mathfrak{w}_{r}^{2}\right) x-\mathfrak{w}_{i}\right)\right) Z_{F, i} \\
& +\frac{\mathfrak{w}_{r}}{2 x a_{1}^{2}\left(x^{3}-1\right)^{2}}\left(a_{1}^{2} a_{3}^{2}\left(x^{2}+x+1\right) \tilde{V}+4 a_{3}^{2} \mathfrak{w}_{i} x \alpha^{2}-2 a_{1}^{2}\left(x^{2}+x+1\right)^{2}\left(2 \mathfrak{w}_{i} x-1\right)\right) Z_{F, r} .
\end{aligned}
$$

The boundary conditions are

$$
Z_{F, r}=x^{4}+\mathcal{O}\left(x^{5}\right), \quad Z_{F, i}=-\mathfrak{w}_{r} x^{5}+\mathcal{O}\left(x^{6}\right)
$$

as $x \rightarrow 0_{+}$, and

$$
Z_{F, r}=z_{f, r, 0}^{h}+\mathcal{O}(y), \quad Z_{F, i}=z_{f, i, 0}^{h}+\mathcal{O}(y),
$$

as $y \equiv 1-x \rightarrow 0_{+}$. Now, there is a clear distinction between the ordered and the disordered phases, even in the limit $b \rightarrow-\infty$. Indeed, the QNM equations (80) and (81) contain $\partial^{2} \tilde{V}$ (set in bold), and

$$
\lim _{b \rightarrow-\infty} \partial^{2} \tilde{V}=\lim _{b \rightarrow-\infty}\left(4+12 b \chi^{2}\right)=4-12\left(f_{[1]}(x)\right)^{2},
$$

where we used (29). In the ordered phase $f_{[1]}(x)$ is a nontrivial function, see Fig. 1, while in the disordered phase it vanishes identically. We present the spectrum of the quasinormal modes on this branch for $b=-4$ as black dots in Fig. 7.

There is a nonpropagating subbranch of the $\mathcal{B} \mathcal{R}_{\text {hair }}$ branch; we call it $\mathcal{B} \mathcal{R}_{\text {hair }}^{\operatorname{Im}}$. The QNM equation of motion on this subbranch is a consistent truncation of Eqs. (80) and (81) with

$$
\mathfrak{w}_{r}=0, \quad Z_{F, i} \equiv 0,
$$

resulting in

$$
\begin{aligned}
0= & Z_{F, r}^{\prime \prime}-\frac{a_{3}^{2} \tilde{V}-2\left(x^{2}+x+1\right)\left(2 \mathfrak{w}_{i} x+x-1\right)}{2\left(x^{3}-1\right) x} Z_{F, r}^{\prime}+\frac{2 \mathfrak{w}_{r}}{x-1} Z_{F, i}^{\prime}+\frac{1}{4 a_{1}^{2} x^{2}\left(x^{3}-1\right)^{2}} \\
& \times\left(4 a_{3}^{2} a_{1}^{2}\left(x^{3}-1\right) \partial^{2} \tilde{V}-4 \chi^{\prime} a_{3}^{2} a_{1}^{2} x\left(x^{3}-1\right) \partial \tilde{V}+a_{3}^{2} a_{1}^{2} x\left(x^{2}+x+1\right)\left(\left(\chi^{\prime}\right)^{2} x(x-1)\right.\right. \\
& \left.-2 \mathfrak{w}_{i}\right) \tilde{V}-4 a_{3}^{2} x^{2}\left(\mathfrak{w}_{i}^{2}-\mathfrak{w}_{r}^{2}\right) \alpha^{2}+4 a_{1}^{2}\left(x^{2}+x+1\right)\left(4 a_{3}^{2}(x-1)+x\left(x^{2}+x+1\right)\right. \\
& \left.\left.\times\left(\left(\mathfrak{w}_{i}^{2}-\mathfrak{w}_{r}^{2}\right) x-\mathfrak{w}_{i}\right)\right)\right) Z_{F, r}-\frac{\mathfrak{w}_{r}}{2 x a_{1}^{2}\left(x^{3}-1\right)^{2}}\left(a_{1}^{2} a_{3}^{2}\left(x^{2}+x+1\right) \tilde{V}+4 a_{3}^{2} \mathfrak{w}_{i} x \alpha^{2}\right. \\
& \left.-2 a_{1}^{2}\left(x^{2}+x+1\right)^{2}\left(2 \mathfrak{w}_{i} x-1\right)\right) Z_{F, i} .
\end{aligned}
$$

Solving (86) with the boundary conditions for $Z_{F, r}$ as in (82) and (83), we find two quasinormal modes: one with $\mathfrak{w}_{i}=\operatorname{Im}[\mathfrak{w}]<0$ and the other one with $\mathfrak{w}_{i}=\operatorname{Im}[\mathfrak{w}]>0$ —these are (correspondingly) the green and the red dots presented for $b=-4$ in Fig. 7. The red dot QNM is what we called $\mathfrak{w}_{u}$ in Sec. I; it is signaling perturbative instability of the hairy black brane horizon, dual to a holographic conformal order. 
In the rest of this section we focus on the $\mathcal{B} \mathcal{R}_{\text {hair }}^{\operatorname{Im}}$ branch. First, we identify the $\mathcal{B} \mathcal{R}_{\text {hair }}^{\operatorname{Im}}$ branch at $\mathfrak{q} \neq 0$. Using the decomposition (73), we find that the truncation

$$
\mathfrak{w}_{r}=0, \quad Z_{F, i} \equiv 0, \quad Z_{H_{i}} \equiv 0
$$

is a consistent one, even for $\mathfrak{q} \neq 0$ :

$$
\begin{aligned}
& 0=Z_{F, r}^{\prime \prime}+\mathcal{A}_{F}^{\operatorname{Im}} Z_{F, r}^{\prime}+\mathcal{B}_{F}^{\operatorname{Im}} Z_{H, r}^{\prime}+\mathcal{C}_{F}^{\operatorname{Im}} Z_{F, r}+\mathcal{D}_{F}^{\operatorname{Im}} Z_{H, r}, \\
& 0=Z_{H, r}^{\prime \prime}+\mathcal{A}_{H}^{\operatorname{Im}} Z_{H, r}^{\prime}+\mathcal{B}_{H}^{\operatorname{Im}} Z_{H, r}+\mathcal{C}_{H}^{\operatorname{Im}} Z_{F, r},
\end{aligned}
$$

with

$$
\begin{aligned}
& \mathcal{A}_{F}^{\operatorname{Im}}=-\frac{a_{3}^{2} \tilde{V}-2\left(x^{2}+x+1\right)\left(2 \mathfrak{w}_{i} x+x-1\right)}{2\left(x^{3}-1\right) x}, \\
& \mathcal{B}_{F}^{\operatorname{Im}}=-\frac{2 a_{3}^{2} \mathfrak{w}_{i}^{2}\left(\chi^{\prime} x \tilde{V}-2 \partial \tilde{V}\right)}{\left(a_{1}^{2} \mathfrak{q}^{2} x^{2}\left(\chi^{\prime}\right)^{2}\left(x^{3}-1\right)+2 a_{1}^{2} \mathfrak{q}^{2}\left(a_{3}^{2} \tilde{V}+2 x^{3}-2\right)-16 \mathfrak{w}_{i}^{2}\right)\left(x^{3}-1\right) x}, \\
& \mathcal{C}_{F}^{\operatorname{Im}}=-\frac{1}{2}\left(a_{3}^{2} a_{1}^{4} \partial \tilde{V} \mathfrak{q}^{2} x^{3}\left(x^{3}-1\right)^{2}\left(\chi^{\prime}\right)^{3}+a_{1}^{2} x^{2}\left(x^{3}-1\right)\left(-2 a_{3}^{2} a_{1}^{2} \mathfrak{q}^{2}\left(x^{3}-1\right) \partial^{2} \tilde{V}+a_{3}^{2}\left(a_{1}^{2} \mathfrak{q}^{2}\right.\right.\right. \\
& \left.\times\left(x^{2}+x+1\right)\left(\mathfrak{w}_{i} x-8 x+8\right)+8 \mathfrak{w}_{i}^{2}\right) \tilde{V}-2 \mathfrak{q}^{2} a_{3}^{2} x^{2}\left(a_{1}^{2} \mathfrak{q}^{2}\left(x^{3}-1\right)-\mathfrak{w}_{i}^{2}\right) \alpha^{2}-2 a_{1}^{2} \mathfrak{q}^{2} \\
& \left.\times\left(x^{2}+x+1\right)\left(x \mathfrak{w}_{i}\left(x^{2}+x+1\right)\left(\mathfrak{w}_{i} x-1\right)+4 a_{3}^{2}(x-1)\right)\right)\left(\chi^{\prime}\right)^{2}+2 a_{3}^{2} a_{1}^{2} \partial \tilde{V} x\left(x^{3}-1\right) \\
& \times\left(a_{1}^{2} \mathfrak{q}^{2}\left(a_{3}^{2} \tilde{V}+10 x^{3}-10\right)-16 \mathfrak{w}_{i}^{2}\right) \chi^{\prime}-4 a_{3}^{2} a_{1}^{2}\left(x^{3}-1\right)\left(a_{1}^{2} \mathfrak{q}^{2}\left(a_{3}^{2} \tilde{V}+2 x^{3}-2\right)-8 \mathfrak{w}_{i}^{2}\right) \\
& \times \partial^{2} \tilde{V}-4 a_{3}^{2} x^{2}\left(a_{1}^{2} \mathfrak{q}^{2}\left(x^{3}-1\right)-\mathfrak{w}_{i}^{2}\right)\left(a_{1}^{2} \mathfrak{q}^{2}\left(a_{3}^{2} \tilde{V}+2 x^{3}-2\right)-8 \mathfrak{w}_{i}^{2}\right) \alpha^{2}-4 a_{1}^{2} a_{3}^{2} \\
& \times\left(x^{2}+x+1\right)\left(a_{1}^{2} \mathfrak{q}^{2}\left(x^{2} \mathfrak{w}_{i}\left(x^{2}+x+1\right)\left(\mathfrak{w}_{i}-1\right)+4 a_{3}^{2}(x-1)\right)+4 \mathfrak{w}_{i}^{3} x\right) \tilde{V}+2 a_{1}^{4} a_{3}^{4} \mathfrak{q}^{2} \mathfrak{w}_{i} \\
& \times x\left(x^{2}+x+1\right) \tilde{V}^{2}-8 a_{1}^{2}\left(x^{2}+x+1\right)\left(x \mathfrak{w}_{i}\left(x^{2}+x+1\right)\left(\mathfrak{w}_{i} x-1\right)+4 a_{3}^{2}(x-1)\right)\left(a_{1}^{2} \mathfrak{q}^{2}\right. \\
& \left.\left.\times\left(x^{3}-1\right)-4 \mathfrak{w}_{i}^{2}\right)\right)\left(a_{1}^{2} x^{2}\left(x^{3}-1\right)^{2}\left(a_{1}^{2} \mathfrak{q}^{2} x^{2}\left(\chi^{\prime}\right)^{2}\left(x^{3}-1\right)+2 a_{1}^{2} \mathfrak{q}^{2}\left(a_{3}^{2} \tilde{V}+2 x^{3}-2\right)-16 \mathfrak{w}_{i}^{2}\right)\right)^{-1} \text {, } \\
& \mathcal{D}_{F}^{\operatorname{Im}}=\frac{1}{4}\left(\left(\chi^{\prime}\right)^{2} x^{2}\left(x^{3}-1\right)+2 a_{3}^{2} \tilde{V}+4\left(x^{2}+x+1\right)\left(2 \mathfrak{w}_{i} x-3 x+3\right)\right)\left(2 \partial \tilde{V}-\chi^{\prime} x \tilde{V}\right) \\
& \times \mathfrak{w}_{i}^{2} a_{3}^{2}\left(\left(a_{1}^{2} \mathfrak{q}^{2} x^{2}\left(\chi^{\prime}\right)^{2}\left(x^{3}-1\right)+2 a_{1}^{2} \mathfrak{q}^{2}\left(a_{3}^{2} \tilde{V}+2 x^{3}-2\right)-16 \mathfrak{w}_{i}^{2}\right)\left(x^{3}-1\right)^{2} x^{2}\right)^{-1}, \\
& \mathcal{A}_{H}^{\mathrm{Im}}=-\frac{1}{2}\left(-a_{1}^{2} \mathfrak{q}^{2} x^{4}\left(x^{3}-1\right)^{2}\left(\chi^{\prime}\right)^{4}-a_{1}^{2} \mathfrak{q}^{2} x^{2}\left(x^{3}-1\right)\left(a_{3}^{2} \tilde{V}+2\left(x^{2}+x+1\right)\left(2 \mathfrak{w}_{i} x-3 x\right.\right.\right. \\
& +3))\left(\chi^{\prime}\right)^{2}-16 \mathfrak{w}_{i}^{2}\left(a_{3}^{2} \tilde{V}-2\left(x^{2}+x+1\right)\left(2 \mathfrak{w}_{i} x+x-1\right)\right)+2 a_{1}^{2}\left(a_{3}^{4} \tilde{V}^{2}-4 \tilde{V}\left(x^{2}+x+1\right)\right. \\
& \left.\left.\times\left(\mathfrak{w}_{i} x+x-1\right) a_{3}^{2}-4(x-1)\left(x^{2}+x+1\right)^{2}\left(2 \mathfrak{w}_{i} x-5 x+5\right)\right) \mathfrak{q}^{2}\right)\left(\left(a_{1}^{2} \mathfrak{q}^{2} x^{2}\left(\chi^{\prime}\right)^{2}\left(x^{3}-1\right)\right.\right. \\
& \left.\left.+2 a_{1}^{2} \mathfrak{q}^{2}\left(a_{3}^{2} \tilde{V}+2 x^{3}-2\right)-16 \mathfrak{w}_{i}^{2}\right)\left(x^{3}-1\right) x\right)^{-1}, \\
& \mathcal{B}_{H}^{\operatorname{Im}}=\frac{1}{16}\left(a_{1}^{4} \mathfrak{q}^{2} x^{6}\left(x^{3}-1\right)^{3}\left(\chi^{\prime}\right)^{6}+4 a_{1}^{4} \mathfrak{q}^{2} x^{4}\left(x^{3}-1\right)^{2}\left(a_{3}^{2} \tilde{V}+\left(x^{2}+x+1\right)\left(2 \mathfrak{w}_{i} x-5 x\right.\right.\right. \\
& +5))\left(\chi^{\prime}\right)^{4}+4 a_{1}^{2} \mathfrak{q}^{2} x^{2}\left(x^{3}-1\right)\left(a_{3}^{4} a_{1}^{2} \tilde{V}^{2}+2 a_{3}^{2} a_{1}^{2}\left(x^{2}+x+1\right)\left(\mathfrak{w}_{i} x-4 x+4\right) \tilde{V}+4 a_{3}^{2} x^{2}\right. \\
& \left.\times\left(a_{1}^{2} \mathfrak{q}^{2}\left(x^{3}-1\right)-\mathfrak{w}_{i}^{2}\right) \alpha^{2}+4 a_{1}^{2}\left(x^{2}+x+1\right)^{2}\left(\mathfrak{w}_{i}^{2} x^{2}-4 \mathfrak{w}_{i} x^{2}+3 \mathfrak{w}_{i} x+3 x^{2}-6 x+3\right)\right) \\
& \times\left(\chi^{\prime}\right)^{2}+32 a_{3}^{2} x^{2}\left(a_{1}^{2} \mathfrak{q}^{2}\left(x^{3}-1\right)-\mathfrak{w}_{i}^{2}\right)\left(a_{1}^{2} \mathfrak{q}^{2}\left(a_{3}^{2} \tilde{V}+2 x^{3}-2\right)-8 \mathfrak{w}_{i}^{2}\right) \alpha^{2}-16 a_{1}^{2}\left(x^{2}+x\right. \\
& +1)\left(a_{3}^{4} a_{1}^{2} \mathfrak{q}^{2}\left(\mathfrak{w}_{i} x-x+1\right) \tilde{V}^{2}-2 a_{3}^{2}\left(a _ { 1 } ^ { 2 } \mathfrak { q } ^ { 2 } ( x ^ { 2 } + x + 1 ) \left(\mathfrak{w}_{i}^{2} x^{2}+\mathfrak{w}_{i} x^{2}-2 \mathfrak{w}_{i} x-6 x^{2}+12 x\right.\right.\right. \\
& \left.-6)+4 \mathfrak{w}_{i}^{3} x\right) \tilde{V}-4\left(x^{2}+x+1\right)\left(a_{1}^{2} \mathfrak{q}^{2}\left(x^{3}-1\right)\left(\mathfrak{w}_{i}^{2} x^{2}-6 \mathfrak{w}_{i} x^{2}+5 \mathfrak{w}_{i} x+9 x^{2}-18 x+9\right)\right. \\
& \left.\left.\left.+4 \mathfrak{w}_{i}^{3} x-4 \mathfrak{w}_{i}^{4} x^{2}\right)\right)\right)\left(a_{1}^{2} x^{2}\left(x^{3}-1\right)^{2}\left(a_{1}^{2} \mathfrak{q}^{2} x^{2}\left(\chi^{\prime}\right)^{2}\left(x^{3}-1\right)+2 a_{1}^{2} \mathfrak{q}^{2}\left(a_{3}^{2} \tilde{V}+2 x^{3}-2\right)-16 \mathfrak{w}_{i}^{2}\right)\right),
\end{aligned}
$$




$$
\begin{aligned}
\mathcal{C}_{H}^{\mathrm{Im}}= & -\frac{1}{4} a_{1}^{2} \mathfrak{q}^{2}\left(\left(\chi^{\prime}\right)^{2} x^{2}\left(x^{3}-1\right)+2 a_{3}^{2} \tilde{V}-12 x^{3}+12\right)\left(-4 \mathfrak{w}_{i}^{2} x^{3}\left(x^{3}-1\right)\left(\chi^{\prime}\right)^{3}+a_{3}^{2} a_{1}^{2} \partial \tilde{V}\right. \\
& \times \mathfrak{q}^{2} x^{2}\left(x^{3}-1\right)\left(\chi^{\prime}\right)^{2}-8 x\left(x^{3}-1\right)\left(a_{3}^{2} a_{1}^{2} \mathfrak{q}^{2} \tilde{V}-6 \mathfrak{w}_{i}^{2}\right) \chi^{\prime}+2 a_{3}^{2} \partial \tilde{V}\left(a_{1}^{2} \mathfrak{q}^{2}\left(a_{3}^{2} \tilde{V}+2 x^{3}-2\right)\right. \\
& \left.\left.-8 \mathfrak{w}_{i}^{2}\right)\right)\left(\left(a_{1}^{2} \mathfrak{q}^{2} x^{2}\left(\chi^{\prime}\right)^{2}\left(x^{3}-1\right)+2 a_{1}^{2} \mathfrak{q}^{2}\left(a_{3}^{2} \tilde{V}+2 x^{3}-2\right)-16 \mathfrak{w}_{i}^{2}\right)\left(x^{3}-1\right) x^{2} \mathfrak{w}_{i}^{2}\right) .
\end{aligned}
$$

Equations (88) have to be solved subject to the following boundary conditions:

In the UV, i.e., as $x \rightarrow 0_{+}$,

$$
Z_{F, r}=x^{4}+\mathcal{O}\left(x^{5}\right), \quad Z_{H, r}=z_{h, r, 0} x^{3}+\mathcal{O}\left(x^{4}\right) .
$$

In the IR, i.e., as $y \equiv 1-x \rightarrow 0_{+}$,

$$
Z_{F, r}=z_{f, r, 0}^{h}+\mathcal{O}(y), \quad Z_{H, r}=z_{h, r, 0}^{h}+\mathcal{O}(y) .
$$

In Fig. 8 we present $\mathfrak{w}_{u}(\mathfrak{q})$-the dispersion relation of the unstable QNM in the ordered phase at $b=-4$; this is the $\mathfrak{q}$ dependence of the red dot in Fig. 7. Note the existence of the critical momenta

$$
\left.\mathfrak{q}_{c}\right|_{b=-4}=1.548
$$

represented by a vertical dashed line, such that for

$$
\mathfrak{q}>\mathfrak{q}_{c} \Rightarrow \operatorname{Im}[\mathfrak{w}(\mathfrak{q})]<0,
$$

i.e., this QNM becomes stable.

In Fig. 9 we present results for $\mathfrak{w}(\mathfrak{q}=0)$ as a function of $b$ for the QNMs on the $\mathcal{B} \mathcal{R}_{\text {hair }}^{\mathrm{Im}}$ branch. There is always an instability in the holographic conformal order, irrespective of the value of $b$ (the solid red curve). The solid green curve represents the stable QNM. Both QNMs become light in the limit $b \rightarrow b_{\text {crit }, 0}=-\frac{3}{2}$, strongly affecting the sound

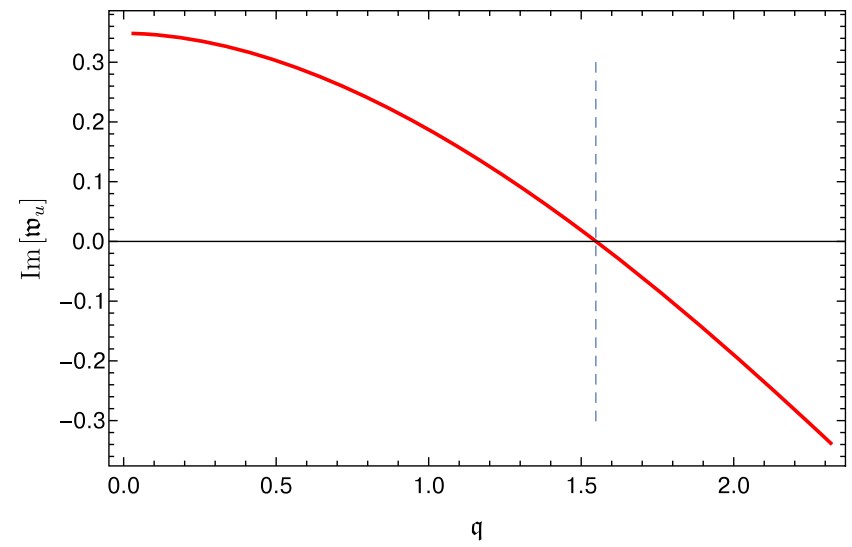

FIG. 8. The dispersion relation $\mathfrak{w}_{u}(\mathfrak{q})$ of the unstable QNM in the ordered phase at $b=-4$. For this mode $\operatorname{Re}\left[\mathfrak{w}_{u}\right]=0$. As typical for the Gregory-Laflamme instability [19], this mode is stabilized for $\mathfrak{q}>\mathfrak{q}_{c}$ [here represented by a vertical dashed line, see Eq. (98)]. waves dispersion; see Sec. III A 3. The dashed horizontal lines represent the $b \rightarrow-\infty$ limit of $\mathfrak{w}$,

$\lim _{b \rightarrow-\infty} \begin{cases}\operatorname{Im}[\mathfrak{w}(0)]=0.461, & \text { unstable QNM }(\text { red }) \\ \operatorname{Im}[\mathfrak{w}(0)]=-0.577, & \text { stable QNM (green) }\end{cases}$

The spectral results (100) are obtained solving Eq. (86) in the limit $b \rightarrow-\infty$. Taking

$Z_{F, r}=Z_{F, 0}+\mathcal{O}\left(\frac{1}{b}\right), \quad \mathfrak{w}_{i}=\mathfrak{w}_{i, 0}+\mathcal{O}\left(\frac{1}{b}\right)$,

and using (29) we find the limiting QNM equation

$$
\begin{aligned}
0= & Z_{F, 0}^{\prime \prime}+\frac{4 \mathfrak{w}_{i, 0} x\left(x^{2}+x+1\right)-12+18 x^{3}}{2 x\left(x^{3}-1\right)} Z_{F, 0}^{\prime} \\
& -\frac{1}{2 x^{2}\left(x^{3}-1\right)^{2}}\left(24\left(x^{3}-1\right) f_{[1]}^{2}-2 x\right. \\
& \times(x-1)\left(x(x+2)\left(x^{2}+x+4\right) \mathfrak{w}_{i, 0}^{2}\right. \\
& \left.\left.+\left(x^{2}+x+1\right)\left(\left(8 x^{2}+7 x+6\right) \mathfrak{w}_{i, 0}+16 x^{2}\right)\right)\right) Z_{F, 0},
\end{aligned}
$$

where we set in bold again the crucial difference between the ordered and the disordered phases:

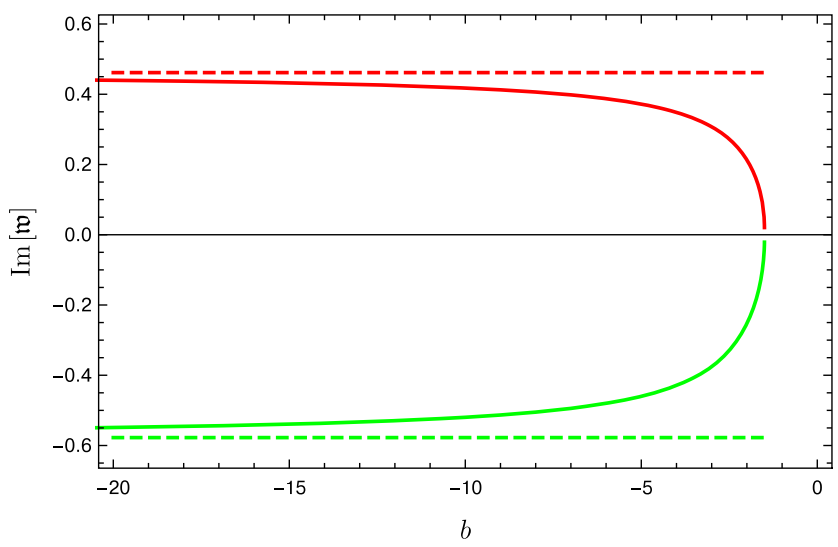

FIG. 9. $\mathfrak{w}(\mathfrak{q}=0)$ of the nonpropagating $(\operatorname{Re}[\mathfrak{w}]=0)$ QNMs on the $\mathcal{B R}_{\text {hair }}^{\operatorname{Im}}$ branch as a function of $b$ in the ordered phase. The dashed horizontal lines represent the limit as $b \rightarrow-\infty$; see Eq. (100). As $b \rightarrow b_{\text {crit }, 0}=-\frac{3}{2}$, these QNMs become light, strongly affecting the dispersion of the sound waves; see Figs. 5-6. 

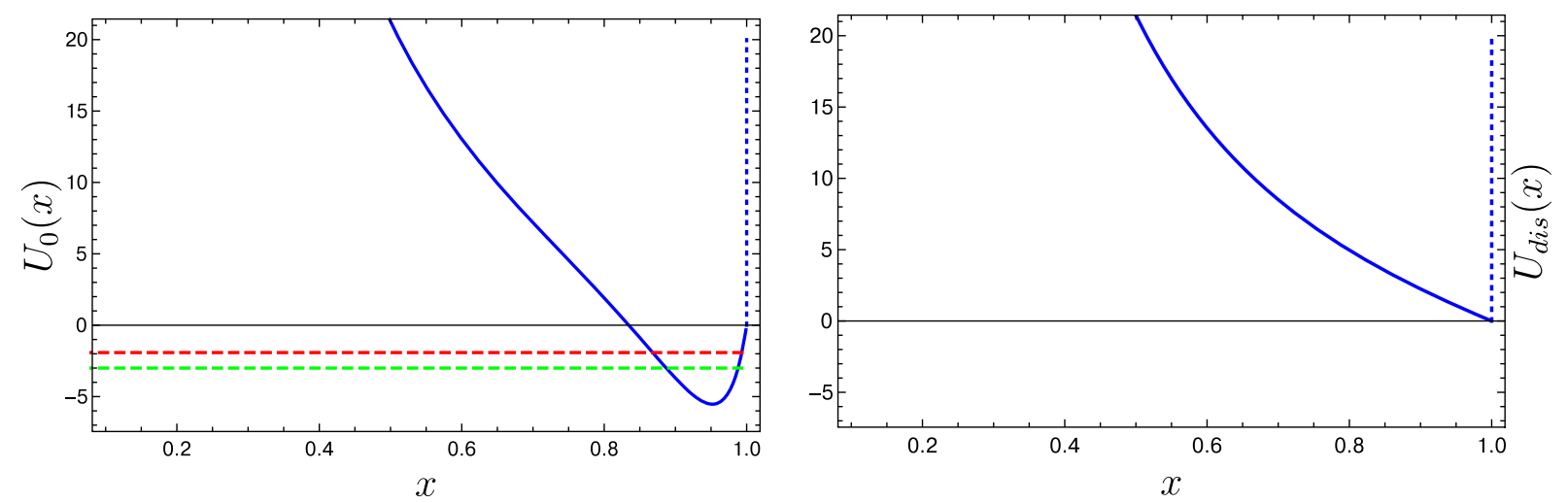

FIG. 10. Existence of the unstable QNM as $b \rightarrow-\infty$ is equivalent to the existence of the bound state in an effective one-dimensional potential (107) with $E<0$. We present this potential, $U_{0}(x)$, for the ordered phase of the overtone 0 (left). The horizontal dashed lines indicate the effective energies of the QNMs (100). In the right panel we present the effective potential in the disordered phase, $U_{\text {dis }}$. Since $U_{\text {dis }}(x) \geq 0$ for $x \in(0,1]$, there cannot be any unstable QNM.

$$
f_{[1]}(x)= \begin{cases}\equiv 0, & \text { in the disordered phase } \\ \text { nontrivial, given by Eq. }(31), & \text { in the ordered phase }\end{cases}
$$

The asymptotic representation (102) explains why the ordered phase is always unstable, while the disordered phase is not. Indeed, introducing a new radial coordinate $u$ with

$$
\frac{d u}{d x}=\frac{1}{1-x^{3}}
$$

and rescaling

$$
Z_{F, 0}=\frac{1}{x^{3}(1-x)^{\mathfrak{w}_{i, 0}}} \Psi_{F}
$$

we obtain a Schrödinger-like equation for $\Psi_{F}$ :

$$
\left[-\frac{d^{2}}{d u^{2}}+U\right] \Psi_{F}=E \Psi_{F}
$$

where the effective $1 \mathrm{~d}$ potential $U$ is

$U(x)=\frac{1-x^{3}}{x^{2}}\left(x^{3}+6-12\left(f_{[1]}\right)^{2}\right), \quad x \in(0,1)$,

and

$$
E=-9 \mathfrak{w}_{i, 0}^{2}
$$

For an unstable QNM, i.e., for $\mathfrak{w}_{i, 0}>0$, the boundary conditions for $Z_{F, 0}$, i.e., $Z_{F, 0} \propto x^{4}$ as $x \rightarrow 0_{+}$and $Z_{F 0} \propto$ $(1-x)^{0}$ as $y=1-x \rightarrow 0_{+}$, imply that given the definition (106),

$$
\Psi_{F} \rightarrow 0, \quad \text { both as } x \rightarrow 0_{+} \text {and } \quad x \rightarrow 1_{-} \text {. }
$$

The potential $U(x)$ of (107) is divergent as $x \rightarrow 0_{+}$, automatically enforcing the first of the boundary conditions in (109); it is vanishing as $x \rightarrow 1_{-}$, so to enforce the second boundary condition in (109) we put an infinite domain wall at $x=1$. Phrased in the language of the effective $1 \mathrm{~d}$ Schrödinger problem with (106) and (108), the presence of the unstable QNM in the spectrum is equivalent to the existence of the bound state in the effective potential (107) with with $E<0$. In Fig. 10 we plot the potential (107) for the ordered phase, i.e., with $f_{[1]}(x) \neq 0$ (the left panel) and for the disordered phase, i.e., with $f_{[1]}(x) \equiv 0$ (the right panel). In the former case the potential dips below zero, allowing for the bound states [represented by the dashed horizontal lines for the QNM frequencies (100)], while in the latter case the potential is always non-negative for $x \in(0,1]$, thus excluding the instability.

We demonstrated above that there is a perturbative instability on an index 0 ordered phase branch/overtone for any value of $b \in\left(-\infty,-\frac{3}{2}\right)$. In fact, there is an instability on the excited branches/overtones of the conformal order. In Fig. 11 we present the spectrum of the QNMs on the $\mathcal{B R}_{\text {hair }}^{\mathrm{Im}}$ branch for the index $i=0,1,2$ background phase overtones in the limit $b \rightarrow-\infty$. These results are obtained solving (102) with the bulk scalar profile function $f_{[1]}$ corresponding to the overtone index $i=0,1,2$; see the left panel of Fig. 1 . Note that the higher branches of the conformal order are more unstable as the value of $\operatorname{Im}\left[\mathfrak{w}_{u}\right]$ increases with the overtone index (the red dots). 


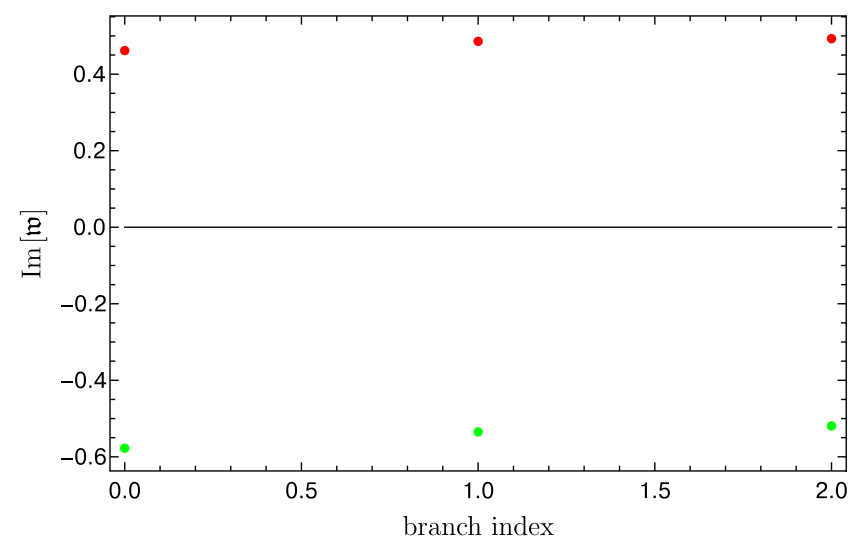

FIG. 11. The spectrum of the QNMs on the $\mathcal{B} \mathcal{R}_{\text {hair }}^{\mathrm{Im}}$ branch at $\mathfrak{q}=0$ and in the limit $b \rightarrow-\infty$ for index $i=0,1,2$ overtones of the holographic conformal order; see Fig. 1. The higher overtones are more unstable as the value of $\operatorname{Im}\left[\mathfrak{w}_{u}\right]$ grows with index (red dots).

\section{NUMERICAL TESTS}

Results reported in this paper involve numerical computation of the QNMs. While some of the conclusions are robust and can be understood in the semianalytic fashion, e.g., the existence of the unstable QNM in each conformal order phase in the limit $b \rightarrow-\infty$ from the effective $1 \mathrm{~d}$ Schrödinger problem, the bulk of the computations involves the heavy numerics. It is thus important to address the question of the numerical tests. We summarize here the implicit and the explicit tests.

(i) Thermal equilibrium phases of the holographic model (2) are conformal. This fact alone predicts the hydrodynamic transport, i.e., the speed of the sound waves $c_{s}$ and the bulk viscosity both in the symmetric and the symmetry broken phases. In Fig. 4 we presented deviations of these quantities, obtained from the QNM computations, from the expected values dictated by the conformal symmetry (9).

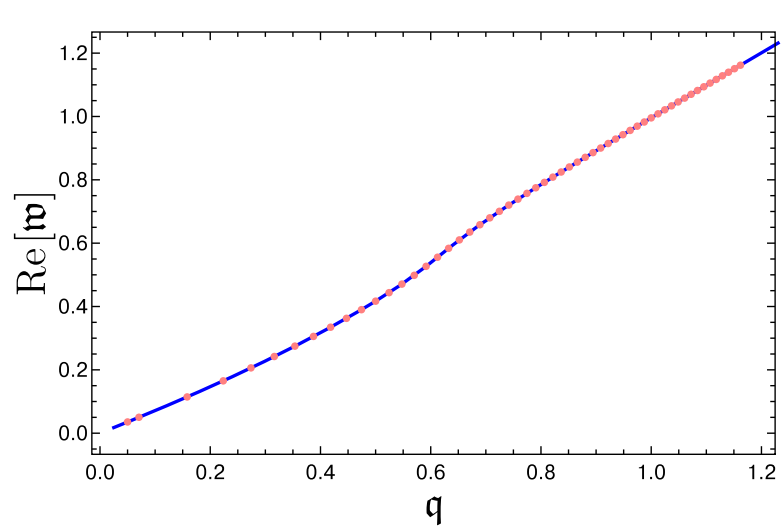

(ii) The universality theorem of [6] implies that the shear viscosity of the holographic conformal order is $s /(4 \pi)$. This result was obtained evaluating the retarded two-point correlation function of the boundary stress-energy tensor in a generic holographic model. In Fig. 3 we presented the deviation of the shear viscosity from the universal result obtained from the dispersion relation of the QNMs in the shear channel.

(iii) In [2] the holographic conformal order was constructed using the background metric ansatz

$$
\begin{aligned}
d s_{4}^{2}= & \frac{\alpha^{2} a(\hat{x})^{2}}{\left(2 \hat{x}-\hat{x}^{2}\right)^{2 / 3}}\left(-(1-\hat{x})^{2} d t^{2}+\left[d x_{1}^{2}+d x_{2}^{2}\right]\right) \\
& +g_{\hat{x} \hat{x}} d \hat{x}^{2}
\end{aligned}
$$

where we denoted a radial coordinate as $\hat{x}$ to distinguish it from the radial coordinate $x$ used here, see (16). The two radial coordinates are related as

$$
x=\left(2 \hat{x}-\hat{x}^{2}\right)^{1 / 3} .
$$

Of course, the results should not depend whether we use $x$ or $\hat{x}$ as a radial coordinate. We emphasize though that the two computational frameworks are very different; this is particularly profound in the computation of the spectrum of the QNMs. Indeed, while the incoming-wave boundary condition for a typical gauge-invariant fluctuation $Z$ is $Z(x) \propto(1-x)^{-i \mathfrak{w}}$, the same boundary condition takes the form

$$
Z(\hat{x}) \propto(1-\hat{x})^{-2 i w}
$$

Since generically the dispersion relations $\mathfrak{w}(\mathfrak{q})$ are complex, the $\mathrm{Re}-\mathrm{Im}$ part splits as in (73) are different for the same normalizations of the wave functions [as in (74)]:

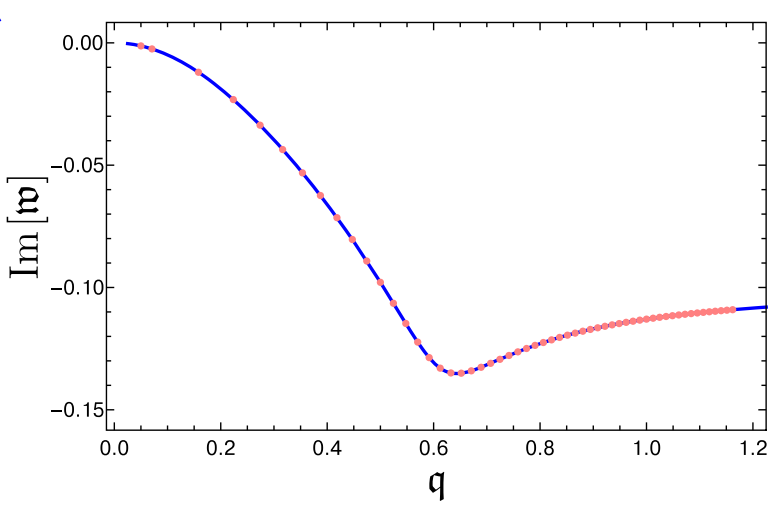

FIG. 12. Comparison of the sound waves dispersion in the ordered phase at $b=-5$ extracted using the $x$ radial coordinate (solid curves) and the $\hat{x}$ radial coordinate, see (111) (the dots). 

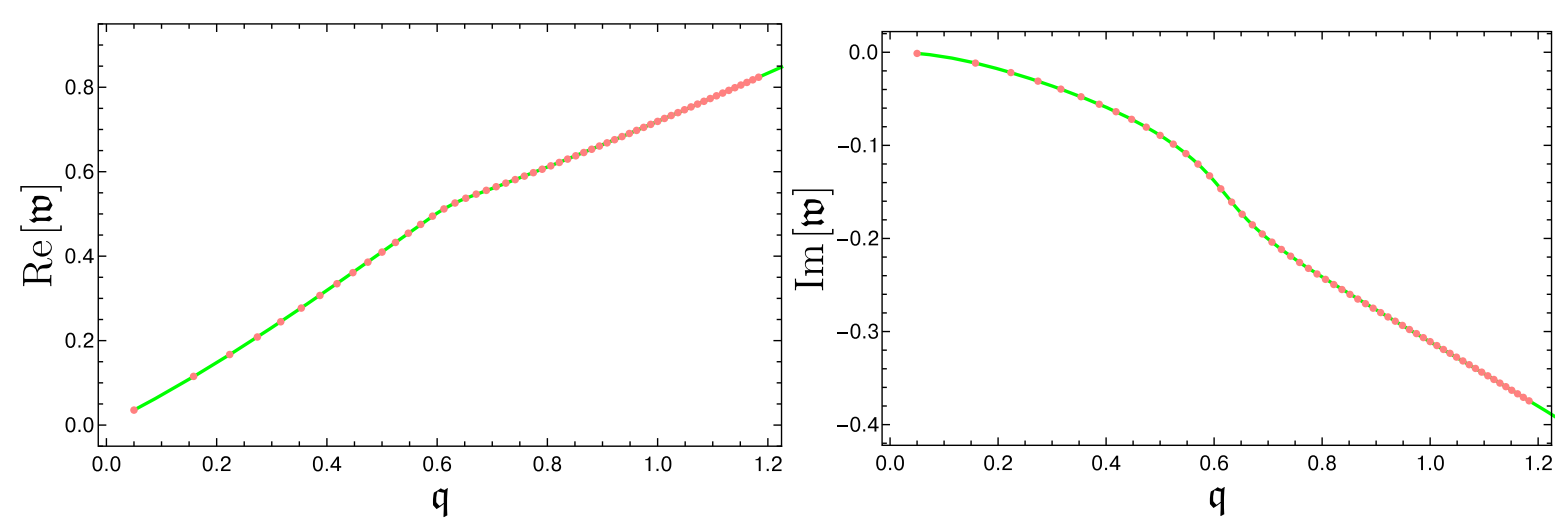

FIG. 13. Comparison of the sound waves dispersion in the ordered phase at $b=-3$ extracted using the $x$ radial coordinate (solid curves) and the $\hat{x}$ radial coordinate, see (111) (the dots).

$$
\operatorname{Re}[Z(x)] \neq \operatorname{Re}[Z(\hat{x})], \quad \operatorname{Im}[Z(x)] \neq \operatorname{Im}[Z(\hat{x})] .
$$

Of course, ultimately, this should not affect the computed spectrum $\mathfrak{w}(\mathfrak{q})$. In Figs. 12 and 13 we compare the spectra of the sound waves computed using the $x$ radial coordinate (solid curves) and using the $\hat{x}$ radial coordinate (dots) in the holographic conformal order at $b=-5$ and $b=-3$ correspondingly. There is an excellent agreement.

(iv) In fact, almost all computations presented in this paper were duplicated in $x$ and $\hat{x}$ radial coordinates.
For example, the fractional difference of the unstable QNM, the red dot in Fig. 7, evaluated in two schemes is $\propto 10^{-9}$.

\section{ACKNOWLEDGMENTS}

Research at Perimeter Institute is supported by the Government of Canada through Industry Canada and by the Province of Ontario through the Ministry of Research and Innovation. This work was further supported by NSERC through the Discovery Grants program.
[1] N. Chai, S. Chaudhuri, C. Choi, Z. Komargodski, E. Rabinovici, and M. Smolkin, Thermal order in conformal theories, Phys. Rev. D 102, 065014 (2020).

[2] A. Buchel, Thermal order in holographic CFTs and no-hair theorem violation in black branes, arXiv:2005.07833.

[3] A. Buchel, SUGRA/Strings like to be bald, arXiv: 2007.09420 .

[4] A. Buchel and C. Pagnutti, Exotic hairy black holes, Nucl. Phys. B824, 85 (2010).

[5] P. K. Kovtun and A. O. Starinets, Quasinormal modes and holography, Phys. Rev. D 72, 086009 (2005).

[6] A. Buchel and J. T. Liu, Universality of the Shear Viscosity in Supergravity, Phys. Rev. Lett. 93, 090602 (2004).

[7] P. Kovtun, D. T. Son, and A. O. Starinets, Viscosity in Strongly Interacting Quantum Field Theories from Black Hole Physics, Phys. Rev. Lett. 94, 111601 (2005).

[8] A. Buchel, On universality of stress-energy tensor correlation functions in supergravity, Phys. Lett. B 609, 392 (2005).

[9] S. S. Gubser and I. Mitra, Instability of charged black holes in anti-de Sitter space, Clay Math. Proc. 1, 221 (2002).

[10] S. S. Gubser and I. Mitra, The evolution of unstable black holes in anti-de Sitter space, J. High Energy Phys. 08 (2001) 018 .
[11] A. Buchel and C. Pagnutti, Correlated stability conjecture revisited, Phys. Lett. B 697, 168 (2011).

[12] A. Buchel and A. Patrushev, Can the correlated stability conjecture be saved?, J. High Energy Phys. 06 (2011) 090.

[13] A. Buchel, On Eling-Oz formula for the holographic bulk viscosity, J. High Energy Phys. 05 (2011) 065.

[14] A. Buchel, U. Gursoy, and E. Kiritsis, Holographic bulk viscosity: GPR versus EO, J. High Energy Phys. 09 (2011) 095.

[15] C. Eling and Y. Oz, A novel formula for bulk viscosity from the null horizon focusing equation, J. High Energy Phys. 06 (2011) 007.

[16] P. Bosch, A. Buchel, and L. Lehner, Unstable horizons and singularity development in holography, J. High Energy Phys. 07 (2017) 135.

[17] A. Buchel, Singularity development and supersymmetry in holography, J. High Energy Phys. 08 (2017) 134.

[18] P. Benincasa, A. Buchel, and A. O. Starinets, Sound waves in strongly coupled non-conformal gauge theory plasma, Nucl. Phys. B733, 160 (2006).

[19] R. Gregory and R. Laflamme, Black Strings and p-Branes are Unstable, Phys. Rev. Lett. 70, 2837 (1993). 